\title{
Geometric study of Lagrangian and Eulerian structures in turbulent channel flow
}

\author{
YUE YANG† AND D. I. PULLIN \\ Graduate Aerospace Laboratories, 205-45, California Institute of Technology, \\ Pasadena, CA 91125, USA \\ (Received 9 August 2010; revised 7 December 2010; accepted 13 December 2010; \\ first published online 2 March 2011)
}

We report the detailed multi-scale and multi-directional geometric study of both evolving Lagrangian and instantaneous Eulerian structures in turbulent channel flow at low and moderate Reynolds numbers. The Lagrangian structures (material surfaces) are obtained by tracking the Lagrangian scalar field, and Eulerian structures are extracted from the swirling strength field at a time instant. The multi-scale and multidirectional geometric analysis, based on the mirror-extended curvelet transform, is developed to quantify the geometry, including the averaged inclination and sweep angles, of both structures at up to eight scales ranging from the half-height $\delta$ of the channel to several viscous length scales $\delta_{v}$. Here, the inclination angle is on the plane of the streamwise and wall-normal directions, and the sweep angle is on the plane of streamwise and spanwise directions. The results show that coherent quasi-streamwise structures in the near-wall region are composed of inclined objects with averaged inclination angle $35^{\circ}-45^{\circ}$, averaged sweep angle $30^{\circ}-40^{\circ}$ and characteristic scale $20 \delta_{v}$, and 'curved legs' with averaged inclination angle $20^{\circ}-30^{\circ}$, averaged sweep angle $15^{\circ}-$ $30^{\circ}$ and length scale $5 \delta_{v}-10 \delta_{v}$. The temporal evolution of Lagrangian structures shows increasing inclination and sweep angles with time, which may correspond to the lifting process of near-wall quasi-streamwise vortices. The large-scale structures that appear to be composed of a number of individual small-scale objects are detected using crosscorrelations between Eulerian structures with large and small scales. These packets are located at the near-wall region with the typical height $0.25 \delta$ and may extend over $10 \delta$ in the streamwise direction in moderate-Reynolds-number, long channel flows. In addition, the effects of the Reynolds number and comparisons between Lagrangian and Eulerian structures are discussed.

Key words: boundary layer structure, turbulence theory, turbulent boundary layers.

\section{Introduction}

Coherent motions or structures with identifiable tube-like shapes that appear to contain concentrated vorticity have been extensively observed and reported from visualizations of laboratory experiments and numerical simulations of wall-bounded turbulence. Although the role played by turbulent coherent, near-wall structures is still not fully understood, over the past several decades many studies have provided evidence supporting the hypothesis that these structures constitute, in some statistical sense, basic elemental vortices that participate in the dynamics of near-wall turbulence 
and are important for drag reduction, turbulent control and other applications (see Robinson 1991; Panton 2001). In addition, it has been argued that the scaling laws and high-order statistics of the mean velocity and velocity fluctuations are influenced by inclined, coherent structures in the near-wall region (see Adrian 2007) and largescale structures in the outer layer (see Hutchins \& Marusic 2007). In what follows, we will generally use the term 'quasi-streamwise vortices', which has been hypothesized in the cited references, to denote individual inclined structures or objects that exist in near-wall turbulence. In the present work, we describe geometry-based metrics that further supports the existence of these structures.

Both Lagrangian- and Eulerian-based approaches have been used to study wall turbulence. Lagrangian methods typically track trajectories of fluid particles, often using visualization techniques. Particle tracers such as hydrogen bubbles (e.g. Kline et al. 1967) or passive scalars such as smoke or dye (e.g. Head \& Bandyopadhyay 1981) show evolving flow structures. These visual studies revealed rich geometries in turbulent structures but remain mainly qualitative (Robinson 1991). Eulerian methodologies benefited from the development of direct numerical simulation (DNS) (e.g. Kim, Moin \& Moser 1987) and experimental particle-image velocimetry (PIV) (e.g. Liu et al. 1991) which provide full, two- or three-dimensional instantaneous velocity fields. The Eulerian structures are usually extracted using either iso-surfaces of vorticity magnitude or popular vortex identification criteria (e.g. Hunt, Wray \& Moin 1988; Chong, Perry \& Cantwell 1990; Jeong \& Hussain 1995).

Major observations on coherent structures in wall turbulence include that the streamwise velocity field close to the wall is organized into alternating narrow streaks of high- and low-speed velocity (Kline et al. 1967) and that candidate hairpin- or $\Lambda$-like vortices may exist in the logarithmic region, while the turbulent motion appears to be less active in the outer layer. The conceptual model of the hairpin vortex was developed by Theodorsen (1952) and supported by experiment (Head \& Bandyopadhyay 1981) and computation visualizations (Moin \& Kim 1982). The modern model of the hairpin vortex is usually described as a combination eddy composed of a hairpin body and two relatively short counter-rotating quasistreamwise vortices that create low-speed streaks in the buffer layer (Adrian 2007). Furthermore, recent DNS and PIV studies provide evidence that hairpin-like structures can autogenerate to form packets that occupy a significant volume fraction of the boundary layer (e.g. Zhou et al. 1999).

Although observation of Eulerian structures can perhaps elucidate turbulence flow physics at a time instant, the Lagrangian approach seems better suited for investigation of the temporal evolution of turbulent coherent structures and their dynamical role in turbulent transition and mixing. This issue was discussed by Green, Rowley \& Haller (2007) who showed the evolution of single vortex-like structures into a packet of similar structures in turbulent channel flow by identifying 'Lagrangian coherent structures' (Haller 2001). Yang, Pullin \& Bermejo-Moreno (2010) illustrated and quantified the evolutionary geometry in the breakdown of initially large-scale Lagrangian structures in isotropic turbulence using a multi-scale geometric analysis.

Consensus on the accepted geometry of vortical structures in wall-bounded turbulence remains elusive. From flow visualization studies of the turbulent boundary layer, Falco (1977) showed that large-scale structures of the smoke concentration field, with typical length scales from $\delta$ to $3 \delta$, inclined to the wall at a characteristic angle $20^{\circ}-25^{\circ}$, while Head \& Bandyopadhyay (1981) measured an inclination angle $40^{\circ}-50^{\circ}$ for candidate hairpins. Using large-eddy simulation (LES) and correlation studies, Moin \& Kim (1985) obtained a most probable inclination angle of local 
vorticity vector as $45^{\circ}$, while Honkan \& Andreopoulos (1997) found that the vorticity is inclined at $35^{\circ}$ from multi-probe hot wire measurements. Using PIV experiments and statistical tools Ganapathisubramani, Longmire \& Marusic (2006) identified individual vortex cores most frequently inclined at $45^{\circ}$. The model developed by Bandyopadhyay (1980) gives that the inclination angle of candidate hairpin packets is $18^{\circ}$. Christensen \& Adrian (2001) found an inclination angle of $12^{\circ}-13^{\circ}$ for the envelope of a series of swirling motions. In contrast, contour-dynamics simulation (Pullin 1981) of a two-dimensional, uniform vorticity layer adjacent to a wall showed inclined structures that resemble flow features observed in the smoke visualization (Falco 1977) of the interface between turbulent and non-turbulent fluid in the outer part of a turbulent boundary layer. This suggests that at least these outer features may not be generated entirely by three-dimensional effects. Open questions remain whose resolution may depend on the scale of structures, Reynolds numbers and the usage of Lagrangian or Eulerian methods in investigations.

The geometry of eddies is also of interest for structure-based models of nearwall turbulence. Predictive models have been developed, based on the attached eddy hypothesis (Townsend 1976), that utilize random superpositions of hierarchies of either hairpins (Perry \& Chong 1982; Perry, Henbest \& Chong 1986; Perry \& Marusic 1995) or hairpin packets (Marusic 2001). A particular geometry or shape of hairpin or $\Lambda$-like vortices is assumed. Additionally, geometrical issues may inform near-wall, subgrid-scale modelling for LES based on small-scale, vortical structures (Chung \& Pullin 2009). The existence of coherent structures with characteristic geometric features may suggest a possible sparse representation for reconstructing a whole channel flow with a greatly reduced number of optimal basis functions utilizing either a wavelet- or curvelet-based extraction method (e.g. Okamoto et al. 2007; Ma et al. 2009).

In the present work, the multi-scale geometric analysis of both Lagrangian and Eulerian structures (Bermejo-Moreno \& Pullin 2008; Yang et al. 2010) in isotropic turbulence is extended to turbulent channel flow by introducing the multi-directional decomposition and mirror-extended data. The extended geometric analysis, which is based on the mirror-extended curvelet transform (see Candès et al. 2006; Demanet \& Ying 2007), will include both multi-scale and multi-directional decompositions of a specific scalar field with non-periodic boundary conditions in wall turbulence. This provides quantitative statistics on the orientation of turbulent structures at different locations, scales and Reynolds numbers. Phenomena in wall turbulence to be investigated include the following: first, the detailed geometry of quasi-streamwise vortices and other structures in the near-wall region (about $5 \delta_{v}$ to $0.3 \delta$ ); second, the structural evolution of near-wall vortices, with initially almost spanwise orientation within the buffer region very close to the wall, into possible $\Lambda$-like or hairpin vortices at a larger wall distance; third, the existence and geometry of packets, based on statistical evidence obtained from multi-scale analysis.

We begin in $\S 2$ by giving a simulation overview for the DNS, using a spectral method, and the computation of Lagrangian fields with the backward-particletracking method. In $\S 3$, a systematic framework is introduced to quantify geometries including averaged inclination and sweep angles of flow structures at multiple scales. Section 4 shows the application of the multi-scale and multi-directional geometric analysis to investigate the geometry of Lagrangian structures at different length scales in time evolution. In $\S 5$ we investigate the geometry of multi-scale, Eulerian structures and provide statistical evidence supporting the formation of structure packets. Finally, some conclusions are drawn in $\S 6$. 


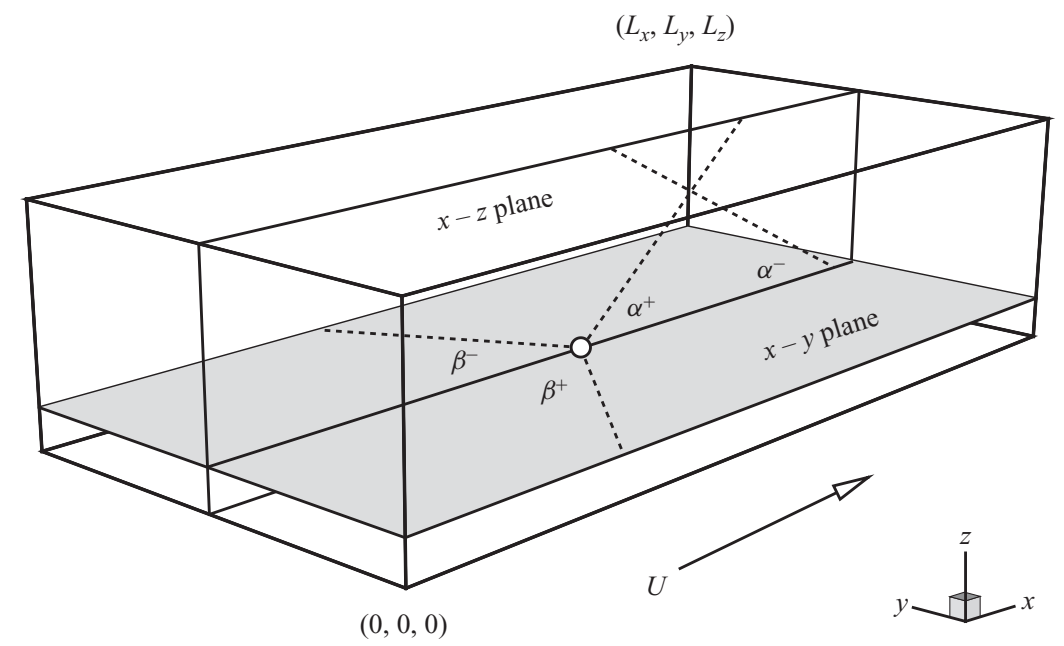

FIGURE 1. Diagram of the computational domain and definition of characteristic angles of structures. Possible structures are sketched by dashed lines.

\section{Simulation overview}

\subsection{Direct numerical simulation}

For turbulent channel flow in a domain with sides $L_{x} \times L_{y} \times L_{z}$ and grids $N_{x} \times N_{y} \times N_{z}$, the incompressible Navier-Stokes equations are non-dimensionalized by the channel half-height $\delta$ and the friction velocity $u_{\tau}$ as

$$
\left.\begin{array}{rl}
\frac{\partial \boldsymbol{u}}{\partial t}+\boldsymbol{u} \cdot \nabla \boldsymbol{u} & =-\nabla p+\boldsymbol{P}_{0}+\frac{1}{R e_{\tau}} \nabla^{2} \boldsymbol{u}, \\
\nabla \cdot \boldsymbol{u} & =0 .
\end{array}\right\}
$$

Here, $p$ denotes the pressure and $R e_{\tau}=u_{\tau} \delta / v$ the Reynolds number, where the friction velocity is $u_{\tau}=\sqrt{\tau_{w} / \rho}$. The viscous, near-wall length scale is $\delta_{v}=v / u_{\tau}$, with wall shear stress $\tau_{w}$, density $\rho$ and kinematic viscosity $\nu$. In the present simulations, we set $\delta=1$ and $u_{\tau} \approx 1$. A constant pressure gradient $\boldsymbol{P}_{0}$ in (2.1) is used to drive the flow. A diagram of the computational domain is shown in figure 1, where $U$ is the mean velocity in the streamwise direction.

The DNS is performed using a spectral method (see Kim et al. 1987): Fourier series in the streamwise $x$ - and spanwise $y$-directions, and the Chebyshev polynomial expansion in the wall-normal $z$-direction. Aliasing errors are removed using the twothirds truncation method. The low-storage third-order semi-implicit Runge-Kutta method (Spalart, Moser \& Rogers 1991) is applied for the temporal discretization and the Courant-Friedrichs-Lewy number

$$
\Delta t \max \left(\frac{\left|u_{x}\right|}{\Delta x}, \frac{\left|u_{y}\right|}{\Delta y}, \frac{\left|u_{z}\right|}{\Delta z}\right)
$$

was set to unity, where $\Delta t$ is the time step size and $\Delta x, \Delta y$ and $\Delta z$ are the mesh sizes in three directions. A summary of DNS parameters is listed in table 1 , where $U_{c}$ is the mean centreline velocity at $z=\delta$ and the superscript ' + ' denotes a non-dimensional quantity scaled by $\delta_{v}$. It is noted that the runs with $L_{x}=2 \pi \delta$ were mainly used to investigate the geometric statistics of structures at intermediate and small scales in the present study. On the other hand, large $L_{x}$ in runs L1 and L2 were chosen for 


\begin{tabular}{lcrrrrrrrrrr}
\hline Run & $R e_{\tau}$ & $L_{x} / \delta$ & $L_{y} / \delta$ & $L_{z} / \delta$ & $N_{x}$ & $N_{y}$ & \multicolumn{1}{c}{$N_{z}$} & $\delta_{v}$ & $U_{c} / u_{\tau}$ & $\Delta x^{+}$ & $\Delta y^{+}$ \\
S1 & 180 & $4 \pi$ & $2 \pi$ & 2 & 192 & 192 & 193 & 0.00556 & 18.28 & 11.77 & 5.89 \\
S2 & 395 & $2 \pi$ & $\pi$ & 2 & 384 & 192 & 193 & 0.00253 & 20.02 & 6.47 & 6.47 \\
S3 & 590 & $2 \pi$ & $\pi$ & 2 & 384 & 384 & 385 & 0.00165 & 21.27 & 9.92 & 4.96 \\
S4 & 950 & $2 \pi$ & $\pi$ & 2 & 768 & 576 & 385 & 0.00105 & 22.68 & 7.77 & 5.18 \\
L1 & 180 & $16 \pi$ & $2 \pi$ & 2 & 768 & 192 & 97 & 0.00556 & 18.15 & 11.77 & 5.89 \\
L2 & 395 & $16 \pi$ & $2 \pi$ & 2 & 1536 & 384 & 193 & 0.00253 & 19.93 & 12.93 & 6.47 \\
L3 & 590 & $8 \pi$ & $\pi$ & 2 & 1152 & 384 & 385 & 0.00165 & 21.16 & 12.87 & 4.96
\end{tabular}

TABLE 1. Summary of DNS parameters.

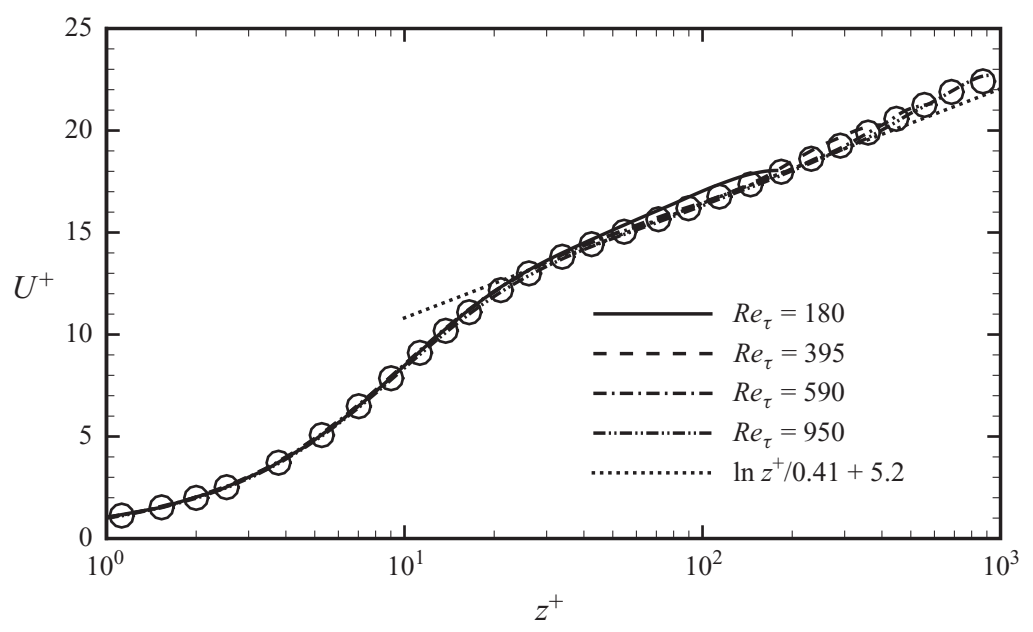

Figure 2. Mean velocity profiles from the DNS. Symbols denote the results at $R e_{\tau}=950$ in del Álamo et al. (2004).

the investigation of hypothesized long structures that may extend over $10 \delta$ in the streamwise direction (e.g. Kim \& Adrian 1999; Jiménez, del Álamo \& Flores 2004; Guala, Hommema \& Adrian 2006). Statistical steady-state, mean velocity profiles for $R e_{\tau}=180,395,590,950$ are shown in figure 2, which agree with the results in Moser, Kim \& Mansour (1999) and del Álamo et al. (2004).

\subsection{Lagrangian field}

The three-dimensional Lagrangian field $\phi(x, t)$ is computed from the scalar convection equation

$$
\frac{\partial \phi}{\partial t}+\boldsymbol{u} \cdot \nabla \phi=0
$$

by the backward-particle-tracking method (see Yang et al. 2010). Here, (2.3) is converted to a set of ordinary differential equations to compute trajectories of fluid particles as

$$
\frac{\partial \boldsymbol{X}\left(\boldsymbol{x}_{0}, t_{0} \mid t\right)}{\partial t}=\boldsymbol{V}\left(\boldsymbol{x}_{0}, t_{0} \mid t\right)=\boldsymbol{u}\left(\boldsymbol{X}\left(\boldsymbol{x}_{0}, t_{0} \mid t\right), t\right),
$$

where $\boldsymbol{X}\left(\boldsymbol{x}_{0}, t_{0} \mid t\right)$ is the location at time $t$ of the fluid particle which was located at $\boldsymbol{x}_{0}$ at the initial time $t_{0}$, and $\boldsymbol{V}\left(\boldsymbol{x}_{0}, t_{0} \mid t\right)$ is the velocity at time $t$ of the fluid particle. First, the Navier-Stokes equations (2.1) are solved numerically on the $N_{x} \times N_{y} \times N_{z}$ 
grid in some time interval from $t_{0}$ to $t>t_{0}$, and the full Eulerian velocity field is saved on disk in this simulation period. The time increment is selected to capture the finest resolved scales in the velocity field. At time $t$ at the end of the solution period, particles are placed at the uniform grid points of $N_{x}^{L} \times N_{y}^{L} \times N_{z}^{L}$. Presently, the resolution of the Lagrangian field is two times that of the velocity field in order to capture fine-scale Lagrangian structures in the evolution (Yang et al. 2010). Then, particles are released and their trajectories calculated by solving (2.4) backwards in time. A three-dimensional, fourth-order Lagrangian interpolation scheme was used to calculate fluid velocity at the particle location. The trajectory of each particle was then obtained using an explicit, second-order Adams-Bashforth scheme. For each particle the backward tracking is performed from $t$ to the initial time $t_{0}$ with the reversed Eulerian velocity fields saved previously. After the backward tracking, initial locations of particles $\boldsymbol{x}_{0}$ can be obtained. From a given initial condition consisting of a smooth Lagrangian field $\phi\left(\boldsymbol{x}_{0}, t_{0}\right)$, we can then obtain $\phi(\boldsymbol{x}, t)$ on the Cartesian grid by a simple mapping with Lagrangian coordinates

$$
\phi(\boldsymbol{x}, t)=\phi\left(X\left(\boldsymbol{x}_{0}, t_{0} \mid t\right), t\right) \longleftrightarrow \phi\left(\boldsymbol{x}_{0}, t_{0}\right) .
$$

\section{Multi-scale and multi-directional methodology}

\subsection{Multi-scale and multi-directional filter based on curvelet transform}

When a scalar field has preferential orientations, e.g. streaks in an image, the Fourier transform of the scalar field should have high intensities at some particular localized regions in Fourier space. Thus, the directional decomposition of the scalar field can be obtained by spectral directional/fan filters, which have been used in computer vision, seismology and image compression (e.g. Bamberger \& Smith 1992). Presently, to obtain statistical, geometric information on preferential orientations in a threedimensional field at different scales, we apply a multi-scale directional filter based on the curvelet transform (see Candès et al. 2006, and references therein) to a sequence of two-dimensional plane-cuts and then compute the angular spectrum and corresponding averaged angles.

An arbitrary two-dimensional scalar field $\varphi(x)$ can be represented by the Fourier expansion

$$
\varphi(\boldsymbol{x})=\sum_{\boldsymbol{k}} \hat{\varphi}(\boldsymbol{k}) \mathrm{e}^{\mathrm{i} k \boldsymbol{x}},
$$

where $\boldsymbol{x}=\left(x_{1}, x_{2}\right), \boldsymbol{k}=\left(k_{1}, k_{2}\right)$ and the Fourier coefficient

$$
\hat{\varphi}(\boldsymbol{k})=\frac{1}{2 \pi} \int \varphi(\boldsymbol{x}) \mathrm{e}^{-\mathrm{i} \boldsymbol{k} \cdot \boldsymbol{x}} \mathrm{d} \boldsymbol{x} .
$$

In the numerical implementation, $\varphi\left(x_{1}, x_{2}\right)$ is discretized on a rectangular domain of side $L_{1} \times L_{2}$ using an $N_{1} \times N_{2}$ grid with indices $\left(n_{1}, n_{2}\right)$ in physical space. The corresponding Fourier space can be discretized on the grid $N_{1} \times N_{2}$ with indices $\left(m_{1}, m_{2}\right)$. The discrete Fourier transform (DFT) and inverse DFT of $\varphi(\boldsymbol{x})$ are

$$
\begin{gathered}
\hat{\varphi}\left(k_{1, m_{1}}, k_{2, m_{2}}\right)=\frac{1}{N_{1}} \frac{1}{N_{2}} \sum_{n_{1}=0}^{N_{1}-1} \sum_{n_{2}=0}^{N_{2}-1} \varphi\left(x_{1, n_{1}}, x_{2, n_{2}}\right) \exp \left[-\mathrm{i}\left(k_{1, m_{1}} x_{1, n_{1}}+k_{2, m_{2}} x_{2, n_{2}}\right)\right], \\
\varphi\left(x_{1, n_{1}}, x_{2, n_{2}}\right)=\sum_{m_{1}=0}^{N_{1}-1} \sum_{m_{2}=0}^{N_{2}-1} \hat{\varphi}\left(k_{1, m_{1}}, k_{2, m_{2}}\right) \exp \left[\mathrm{i}\left(k_{1, m_{1}} x_{1, n_{1}}+k_{2, m_{2}} x_{2, n_{2}}\right)\right],
\end{gathered}
$$


respectively, with

$$
k_{i, m_{i}}=m_{i} \Delta k_{i}, \quad x_{i, n_{i}}=n_{i} \Delta x_{i}, \quad \Delta x_{i}=L_{i} / N_{i}, \quad \Delta k_{i}=2 \pi / L_{i} .
$$

A filtered $\varphi(\boldsymbol{x})$ at scale $j$ and along the direction $l$ can then be extracted from $\hat{\varphi}(\boldsymbol{k})$ in Fourier space by the frequency window function

$$
U_{j}(r, \theta)=2^{-3 j / 4} W\left(2^{-j} r\right) V\left(t_{l}(\theta)\right),
$$

in polar coordinates $(r, \theta)$ with $r=\sqrt{k_{1}^{2}+k_{2}^{2}}$ and $\theta=\arctan \left(k_{2} / k_{1}\right)$. The frequency window function $U_{j}(r, \theta)$ is based on the curvelet transform (Candès et al. 2006), which is a combination of the radial window function (e.g. Ma et al. 2009)

$$
W(r)= \begin{cases}\cos (\pi \mu(5-6 r) / 2), & 2 / 3 \leqslant r \leqslant 5 / 6, \\ 1, & 5 / 6 \leqslant r \leqslant 4 / 3, \\ \cos (\pi \mu(3 r-4) / 2), & 4 / 3 \leqslant r \leqslant 5 / 3, \\ 0, & \text { else, }\end{cases}
$$

and the angular window function

$$
V\left(t_{l}\right)= \begin{cases}1, & \left|t_{l}\right| \leqslant 1 / 3, \\ \cos \left(\pi \mu\left(3\left|t_{l}\right|-4\right) / 2\right), & 4 / 3 \leqslant t_{l} \leqslant 5 / 3, \\ 0, & \text { else, }\end{cases}
$$

with the smoothing function $\mu(x)=3 x^{2}-2 x^{3}$ satisfying

$$
\mu(x)=\left\{\begin{array}{ll}
1, & x \leqslant 0, \\
0, & x \geqslant 1,
\end{array} \quad \mu(x)+\mu(1-x)=1 .\right.
$$

Both radial and angular window functions satisfy the admissibility conditions:

$$
\begin{gathered}
\sum_{r=-\infty}^{\infty} W^{2}\left(2^{j} r\right)=1, \quad r>0, \\
\sum_{t_{l}=-\infty}^{\infty} V^{2}\left(t_{l}\right)=1, \quad t_{l} \in \mathbb{R} .
\end{gathered}
$$

The frequency window functions in Fourier space are plotted in figure 3. Each is supported on a region bounded by two neighbouring circular wedges in the range of wavenumbers $2^{j-1} \leqslant r \leqslant 2^{j+1}$. The localization of $U_{j}(r, \theta)$ in Fourier space implies that the corresponding spatial structure in physical space is a needle-shaped element (curvelet), whose envelope has the geometric scaling relation (Candès et al. 2006)

$$
\text { length } \approx 2^{-j / 2}, \quad \text { width } \approx 2^{-j} .
$$

From (3.12), the characteristic length scale is $\mathscr{L}_{j} \approx 2^{-j}$ at scale $j$, which is the characteristic width of the 'curvelet' at scale $j$ in physical space. The breakdown of the normalized characteristic length scales of structures after the multi-scale decomposition is given in table 2.

The frequency windows cover the whole Fourier domain at characteristic length scales

$$
2^{-j}, \quad j=-1,0,1,2,3, \ldots,
$$




\begin{tabular}{|c|c|c|c|c|c|c|c|c|c|}
\hline$R e_{\tau}$ & Length & 1 & 2 & 3 & 4 & 5 & 6 & 7 & 8 \\
\hline & $\mathscr{L}_{j} / \delta$ & 0.5 & 0.25 & 0.125 & 0.0625 & 0.0313 & 0.0156 & 0.0078 & 0.0039 \\
\hline 180 & $\mathscr{L}_{i} / \delta_{v}$ & 90.0 & 45.0 & 22.50 & 11.25 & 5.625 & 2.813 & 1.406 & 0.703 \\
\hline 395 & $\mathscr{L}_{j} / \delta_{v}$ & 197.5 & 98.75 & 49.38 & 24.69 & 12.34 & 6.172 & 3.086 & 1.543 \\
\hline 590 & $\mathscr{L}_{j} / \delta_{v}$ & 295.0 & 147.5 & 73.75 & 36.88 & 18.44 & 9.219 & 4.609 & 2.305 \\
\hline 950 & $\mathscr{L}_{i} / \delta_{v}$ & 475.0 & 237.5 & 118.8 & 59.38 & 29.69 & 14.84 & 7.422 & 3.711 \\
\hline
\end{tabular}

TABLE 2. Breakdown of characteristic length scales for the filtered scales $j$ in terms of the half-height $\delta$ and viscous length scale $\delta_{v}$ in turbulent channel flow.

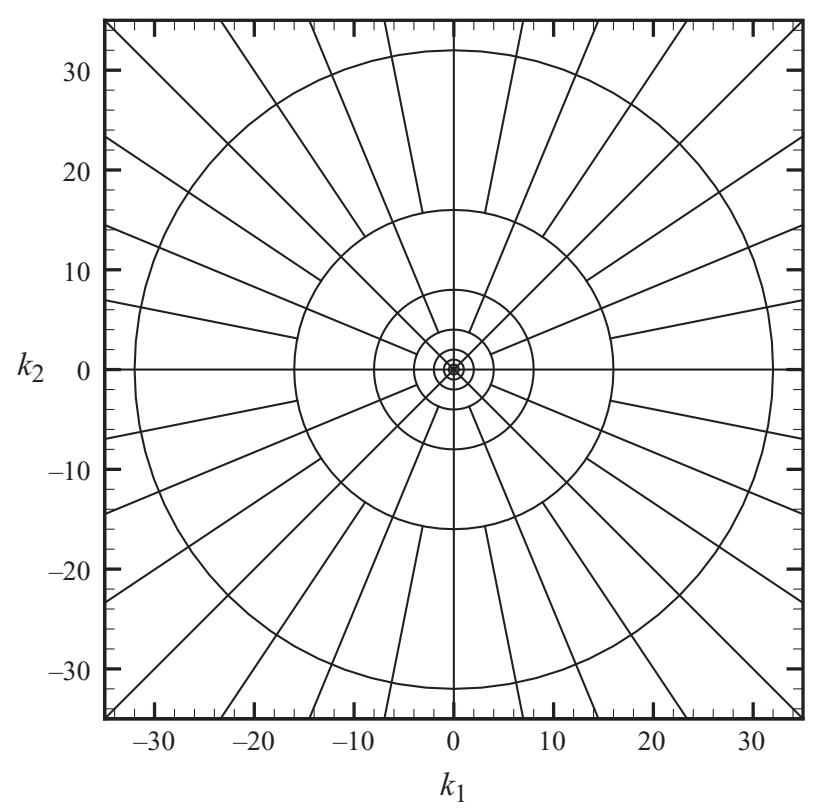

FIGURE 3. Frequency window functions $U_{j}(r, \theta)$ supported on circular wedges in Fourier space.

and the equidistant sequence of rotation angles

$$
\theta_{j, l} \equiv \pi l 2^{-[j / 2\rceil} / 2, \quad l=0,1, \ldots, 4 \cdot 2^{\lceil j / 2\rceil}-1,
$$

where $\lceil x\rceil$ gives the smallest integer greater than or equal to $x$.

\subsection{Multi-scale and multi-directional decomposition}

By applying the radial window function $W(r)$ on the Fourier coefficients $\hat{\varphi}(\boldsymbol{k})$ and then using the inverse Fourier transform as

$$
\varphi_{j}(\boldsymbol{x})=\int \hat{\varphi}(\boldsymbol{k}) W\left(2^{-j} r\right) \mathrm{e}^{\mathrm{i} \boldsymbol{k} \cdot \boldsymbol{x}} \mathrm{d} \boldsymbol{x},
$$

for each scale $j=j_{0}, \ldots, j_{e}$, with $j_{0}=0$ and $j_{e}=\log _{2}\left(\min \left(N_{1} \Delta k_{1}, N_{2} \Delta k_{2}\right) / 2\right)$, we can obtain a multi-scale decomposition of the original scalar field $\varphi(x)$ into a total of $j_{e}-j_{0}+1$ scale-dependent fields. For convenience we will subsequently label scaledependent fields by the index $j=0, \ldots, j_{e}$, with $j=0$ corresponding to the largest scale field and $j=j_{e}$ the smallest resolved-scale field. Subsequently, and according to table $2, \mathscr{L}_{j}>0.2 \delta$ will be referred to as 'large scale', $\mathscr{L}_{j}<10 \delta_{v}$ as 'small scale' and in 

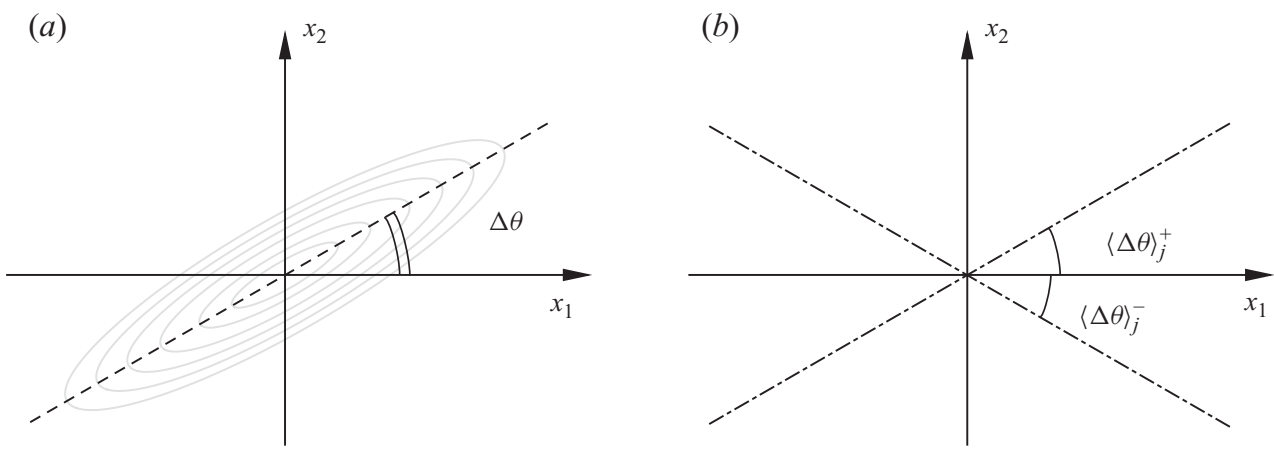

FIGURE 4. Diagram of the deviation angles away from the horizontal axis in physical space. (a) Deviation angle and $(b)$ averaged deviation angles.

between as 'intermediate scale'. The filter for the largest scale is defined as

$$
\left|W_{0}(r)\right|^{2}=1-\sum_{j \geqslant 1}\left|W\left(2^{-j} r\right)\right|^{2} .
$$

The characteristic direction $l^{\prime}$ of $\varphi(x)$ in physical space is orthogonal to that in Fourier space. The discrete deviation angle away from the horizontal axis in physical space

$$
\Delta \theta=2^{-\lceil j / 2\rceil} \frac{\pi l^{\prime}}{2}, \quad l^{\prime}=-2^{\lceil j / 2\rceil}, \ldots,-1,0,1, \ldots, 2^{\lceil j / 2\rceil},
$$

is sketched in figure $4(a)$ for scale $j$. The multi-scale orientation information of $\varphi(\boldsymbol{x})$ can be defined as the normalized angular spectrum

$$
\Phi_{j}(\Delta \theta) \equiv \frac{\int \hat{\varphi}(\boldsymbol{k}) U_{j}(r, \theta) \mathrm{d} \boldsymbol{k}}{\int U_{j}(r, \theta) \mathrm{d} \boldsymbol{k}}, \quad-\frac{\pi}{2} \leqslant \Delta \theta \leqslant \frac{\pi}{2}
$$

at scale $j$ and direction $l$, where $t_{l}=\left(\pi / 2-\theta+\theta_{j, l^{\prime}}\right) / \theta_{j, 1}$ for the angular window function $V\left(t_{l}\right)$ in $U_{j}(r, \theta)$.

The averaged deviation angles away from the horizontal direction are sketched in figure $4(b)$. They can be obtained as

$$
\langle\Delta \theta\rangle_{j}^{+} \equiv \frac{\sum_{l^{\prime}=0}^{l_{\max }^{\prime}} \Phi_{j}(\Delta \theta) \Delta \theta}{\sum_{l^{\prime}=0}^{l_{\max }^{\prime}} \Phi_{j}(\Delta \theta)} \text { and }\langle\Delta \theta\rangle_{j}^{-} \equiv \frac{\sum_{l^{\prime}=l_{\min }^{\prime}}^{0} \Phi_{j}(\Delta \theta) \Delta \theta}{\sum_{l^{\prime}=l_{\min }^{\prime}}^{0} \Phi_{j}(\Delta \theta)}
$$

where $l_{\text {max }}^{\prime}=2^{\lceil j / 2\rceil}$ and $l_{\text {min }}^{\prime}=-2^{\lceil j / 2\rceil}$, which we take to define characteristic angles of a two-dimensional scalar field in the statistical sense. As an example, the scale decomposition of an image of a cross

$$
\varphi\left(x^{\prime}, y^{\prime}\right)=\exp \left(-100 x^{\prime 2}\right)+\exp \left(-100 y^{\prime 2}\right)-\exp \left(-100\left(x^{\prime 2}+y^{\prime 2}\right)\right)
$$

with

$$
x^{\prime}=x \cos 45^{\circ}-y \sin 45^{\circ} \quad \text { and } \quad y^{\prime}=x \sin 45^{\circ}+y \cos 45^{\circ}
$$


(a)

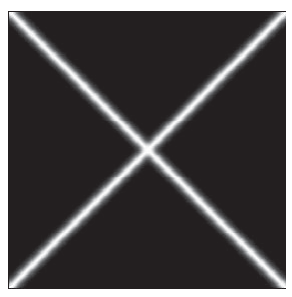

(b)

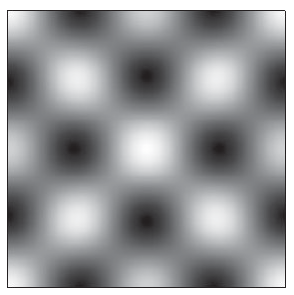

(c)

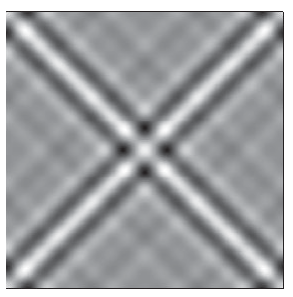

(d)

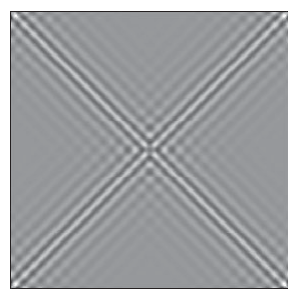

Figure 5. Scale decomposition of an image of the cross. (a) Original, (b) scale 1, $(c)$ scale 3 and $(d)$ scale 5 .
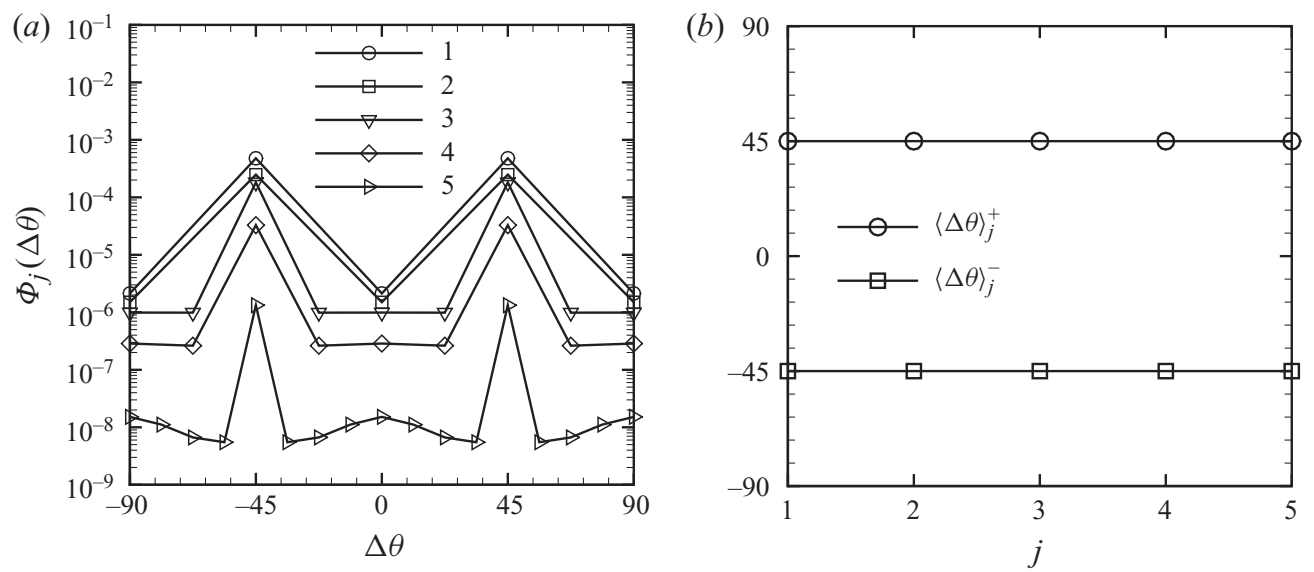

FIGURE 6. Directional decomposition of an image of the cross at different scales. (a) Angular spectrum and (b) average deviation angles (degrees).

is shown in figure 5. The angular spectrum from the directional decomposition and averaged deviation angles defined in (3.19) are shown in figure 6, which show characteristic angles $\pm 45^{\circ}$ of the image.

We remark that the multi-scale and multi-directional decomposition in the present study is based on the continuous curvelet transform rather than the fast discrete curvelet transform (see Candès et al. 2006). This is because we find that using the frequency window functions in the continuous curvelet transform, e.g. (3.7) and (3.8) supported on the circular wedges as shown in figure 3, gives more accurate multiscale angular spectra (3.18) than those from the window functions supported on the sheared trapezoid wedges in the fast discrete curvelet transform algorithm.

When a scalar field has non-periodic boundaries, e.g. $\varphi$ on the $x-z$ plane in channel flows, the DFT in (3.3) may result in numerical-oscillatory artefacts near boundaries. This can be resolved by using the discrete cosine transform instead of the DFT in the direction with non-periodic boundaries. This is equivalent to applying the DFT or the fast Fourier transform (FFT) to the mirror-extended scalar field and corresponds to the mirror-extended curvelet transform (Demanet \& Ying 2007). Presently, before the FFT, we copy and flip the two-dimensional scalar field by the one-dimensional mirror extension in the wall-normal direction with non-periodic boundary conditions as

$$
\left\{\varphi_{1}, \varphi_{2}, \ldots, \varphi_{N-1}, \varphi_{N}\right\} \rightarrow\left\{\varphi_{1}, \varphi_{2}, \ldots, \varphi_{N-1}, \varphi_{N}, \varphi_{N-1}, \ldots, \varphi_{2}\right\}
$$




\section{Lagrangian structures in turbulent channel flow}

\subsection{Geometry of Lagrangian structures on the $x-z$ (streamwise and wall-normal) plane}

By tracking the three-dimensional Lagrangian field $\phi(x, t)$ in turbulent channel flow, Lagrangian structures are extracted as iso-contours of $\phi$ at different times. In this section, we will use a time series of Eulerian velocity fields from runs S1 and S2 (see table 1) for two Reynolds numbers to solve the $\phi$-equation (2.3). It is noted that the investigation of Lagrangian structures is computationally intensive, requiring storage of sequences of time-resolved Eulerian velocity fields for the backwardparticle-tracking method (Yang et al. 2010).

Previous studies showed that the important geometric feature of leaning or slanting vortex structures is that they tend to incline at an angle to the streamwise direction in the logarithmic law region (see Adrian 2007). For studying Lagrangian structures on the $x-z$ plane, we utilize an initial Lagrangian field $\phi_{0} \equiv \phi(x, t=0)=\sin 3 x \sin \pi z$, with length scale equal to the half-height $\delta=1$. To distinguish turbulent dynamics and kinematics (Yang et al. 2010), we choose the largest time $t_{c}=4$ for tracking the Lagrangian field $\phi$ according to the typical period of the 'cyclic' process of the evolving near-wall turbulent structures (see Hinze 1975), where the large-scale, nondimensional time is $t_{c}=t U_{c} / \delta$. Since a large-scale Lagrangian structure is imposed by the initial condition, next we investigate only structures with scales $j \geqslant 3$.

The temporal evolution of the Lagrangian field with colour intensity proportional to $\phi \in[-1,1]$ on the $x-z$ plane in turbulent channel flow at $R e_{\tau}=395$ is shown in figure 7. We can see that the initial regular, periodical blob-like objects are stretched into ramp-shaped structures by the mean shear and small-scale turbulent motions. Unlike the scalar properties for previous smoke visualization in turbulent boundary layer experiments (e.g. Head \& Bandyopadhyay 1981), the Lagrangian scalar field is non-diffusive, so we can visualize and analyse fine structures with a high-resolution grid for $\phi$. In figure 7 the vivid contrast evident between smooth and chaotic regions illustrates the strong intermittency present in the Lagrangian field for the present turbulent channel flow.

As shown in figure 1, we define the inclination angle $\alpha$ between an inclined structure projected on the $x-z$ plane and the $x$-direction. In terms of the variables in the multiscale and multi-directional method as shown in figure 4, we have $\phi\left(x, y=y_{p}, z\right) \leftrightarrow \varphi$, $x \leftrightarrow x_{1}, z \leftrightarrow x_{2}, \alpha^{+} \leftrightarrow\langle\Delta \theta\rangle^{+}$and $\alpha^{-} \leftrightarrow\langle\Delta \theta\rangle^{-}$. Evolution of individual components of the Lagrangian field can be obtained following the scale decomposition of $\phi$ on the $x-z$ plane by (3.15). These are shown, at $R e_{\tau}=395$, for the intermediate scale $j=4$ and small scale $j=6$ in figures 8 and 9 , respectively. The characteristic length scale for each scale index $j$ is quantified in table 2 . Then, the orientation information of $\phi(x, t)$ on the $x-z$ plane at different scales can be obtained by the normalized angular spectra $\Phi_{j}(\Delta \theta)$ defined in (3.18), which are shown in figure 10 at $t_{c}=1$ and $t_{c}=4$. The increasing $\Phi_{j}(\Delta \theta)$ at scales $j \geqslant 3$ shows a cascade process from large scales to small scales for $\phi$ in the temporal evolution. At each time, smaller scale structures appear to show a greater tendency to 'attach' to the wall than larger structures in the sense of the predominance of small deviation angles in $\Phi(\Delta \theta)$ for large $j$.

As shown in figure 10, the angular spectra are almost symmetric for all scales. Therefore, assuming the symmetry of inclined structures with the centreline on the $x-$ $z$ plane in the statistical sense, we define the averaged inclination angle $\langle\alpha\rangle=\left(\left\langle\alpha^{+}\right\rangle+\right.$ $\left.\left\langle\alpha^{-}\right\rangle\right) / 2$. Figure 11( $\left.a\right)$ shows the temporal evolution of $\langle\alpha\rangle$ for Lagrangian structures at intermediate and small scales (also refer figures 8 and 9). Here, the additional 
(a)

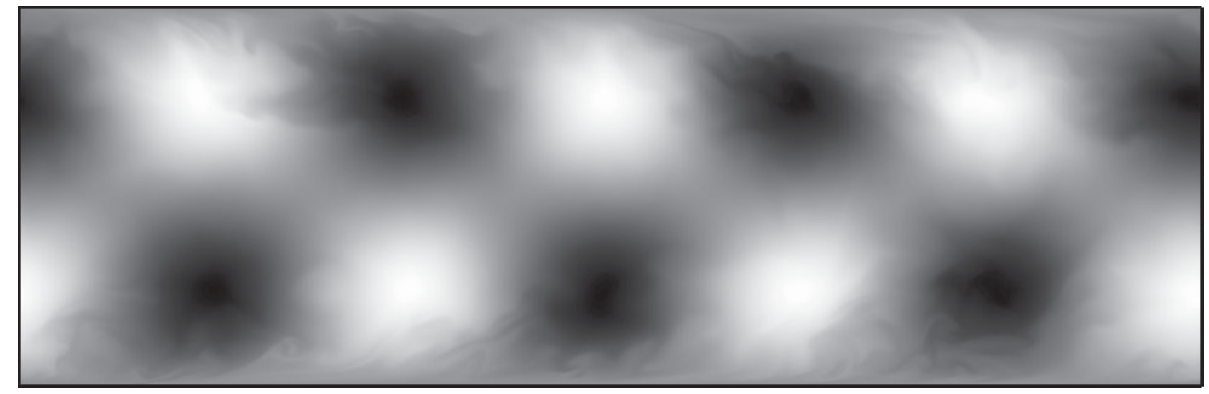

$(b)$

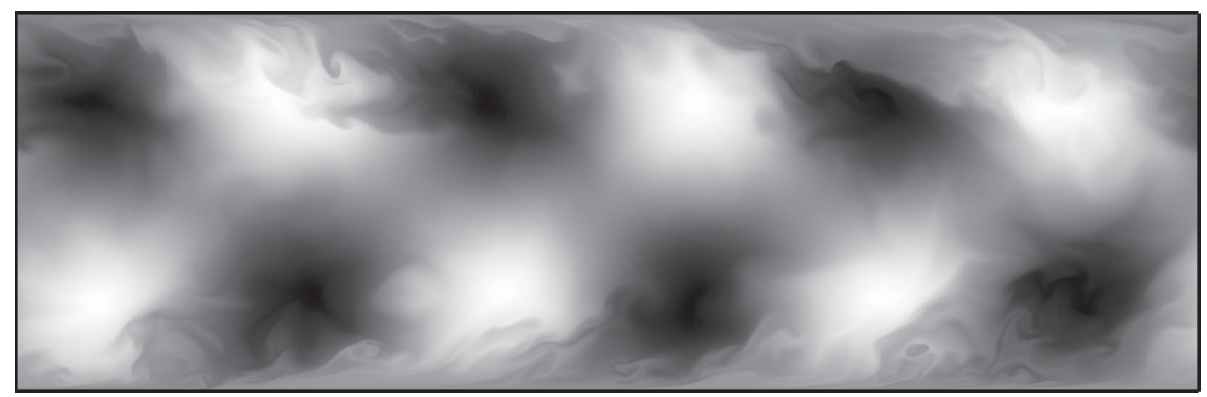

(c)

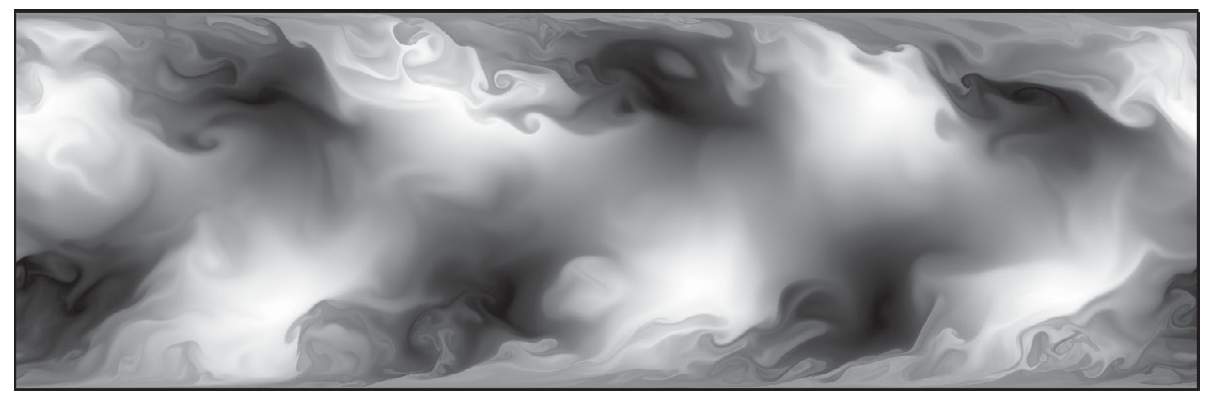

(d)

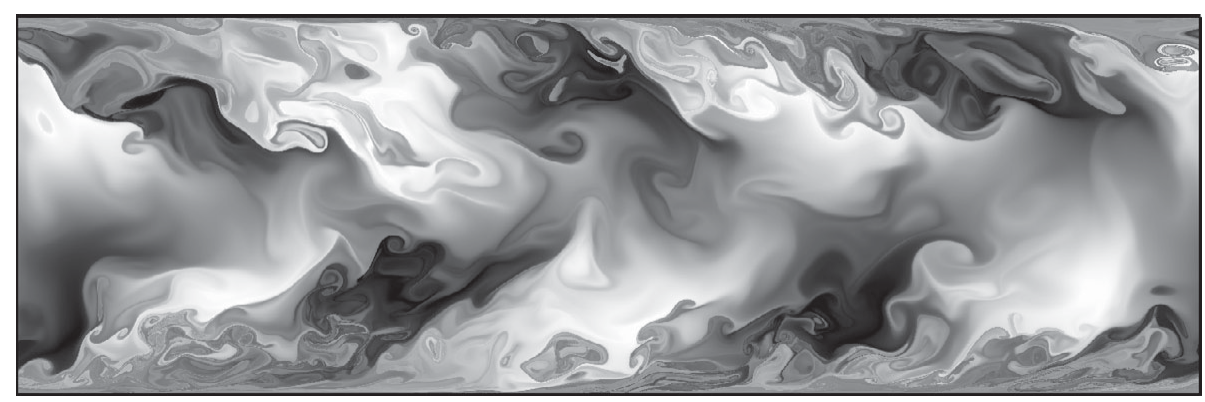

FIGURE 7. Evolution of Lagrangian structures on the $x-z$ plane $(0 \leqslant x \leqslant 2 \pi, 0 \leqslant z \leqslant 2)$ in turbulent channel flow for $R e_{\tau}=395$ (run S2). (a) $t_{c}=0.5$, (b) $t_{c}=1,(c) t_{c}=2$ and $(d) t_{c}=4$. 
(a)

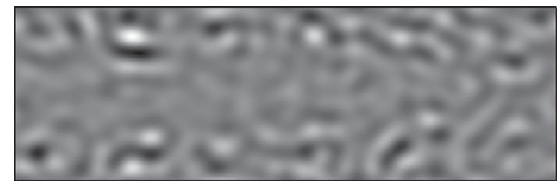

(c)

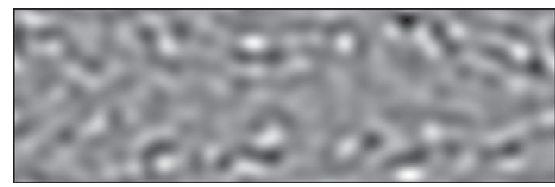

(b)

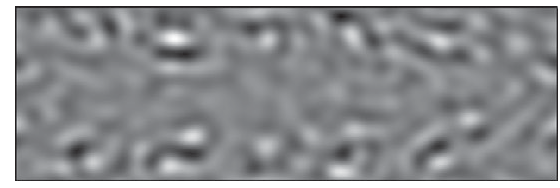

$(d)$

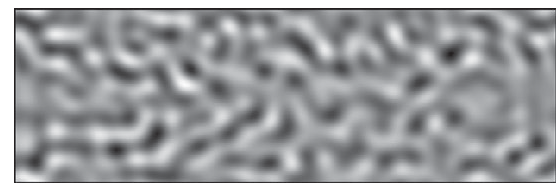

FIGURE 8. Evolution of Lagrangian structures at scale 4 on the $x-z$ plane $(0 \leqslant x \leqslant 2 \pi, 0 \leqslant z \leqslant 2)$ in turbulent channel flow for $R e_{\tau}=395$ (run S2). (a) $t_{c}=0.5$, (b) $t_{c}=1,(c) t_{c}=2$ and $(d) t_{c}=4$.

(a)

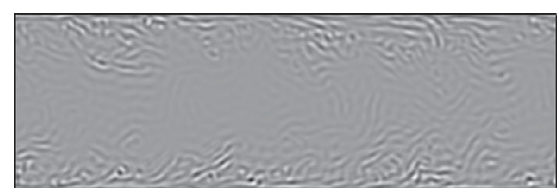

(c)

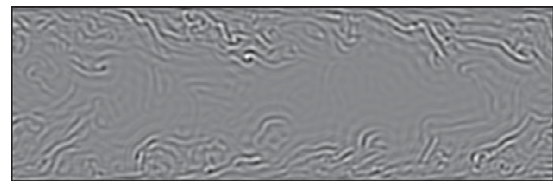

(b)

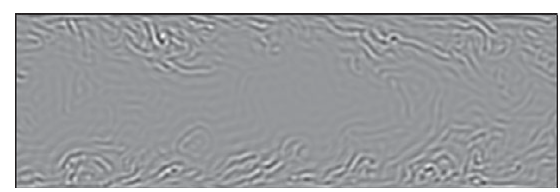

(d)

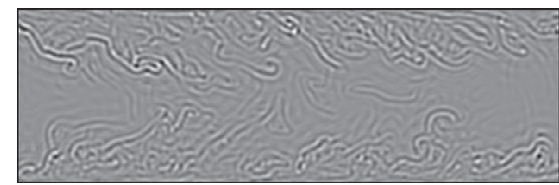

FIGURE 9. Evolution of Lagrangian structures at scale 6 on the $x-z$ plane $(0 \leqslant x \leqslant 2 \pi, 0 \leqslant z \leqslant 2)$ in turbulent channel flow for $R e_{\tau}=395$ (run S2). (a) $t_{c}=0.5,(b) t_{c}=1,(c) t_{c}=2$ and $(d) t_{c}=4$.

averaging on $\langle\alpha\rangle$ was taken over $50 x-z$ planes at $y=y_{p}$ uniformly distributed between $y=0$ and $y=L_{y}$. As shown in figure $9(a)$, the small-scale structures with small $\langle\alpha\rangle$ appear at early times, around $t_{c}=0.5$, produced by intense near-wall shear motions. Then, the small-scale structures are uplifted as shown in figures $9(c)$ and $9(d)$, which may signal ejections of low-speed fluid outward from the wall. Finally, some small-scale structures are bent downwards to the wall, which may be imprints of the sweeps of high-speed fluid towards the wall. In figure 11(a), $\langle\alpha\rangle$ grows with increasing time; the trend is slightly slower for $t_{c}>3$. These observations are consistent with the conceptual ejection-sweep-burst-inrush process (Hinze 1975). At $t_{c}=1$ and $t_{c}=4,\langle\alpha\rangle$ at different scales is shown in figure 11(a). We find that $\langle\alpha\rangle$ of Lagrangian structures at scales smaller than $20 \delta_{v}$ are higher for $R e_{\tau}=395$ than for $R e_{\tau}=180$, which may imply stronger turbulent transport in higher-Reynolds-number flows by coherent motions that eject more fluid from the viscous sublayer to the logarithmic region.

In addition, from simulations using different initial fields, e.g. $\phi_{0}=\sin 3 x$ and $\phi_{0}=\pi z$ (not shown), we find that the resulting Lagrangian structures produce quantitatively similar averaged inclination angles at intermediate scales and small scales for long times to those described above. This suggests that an attractor for 

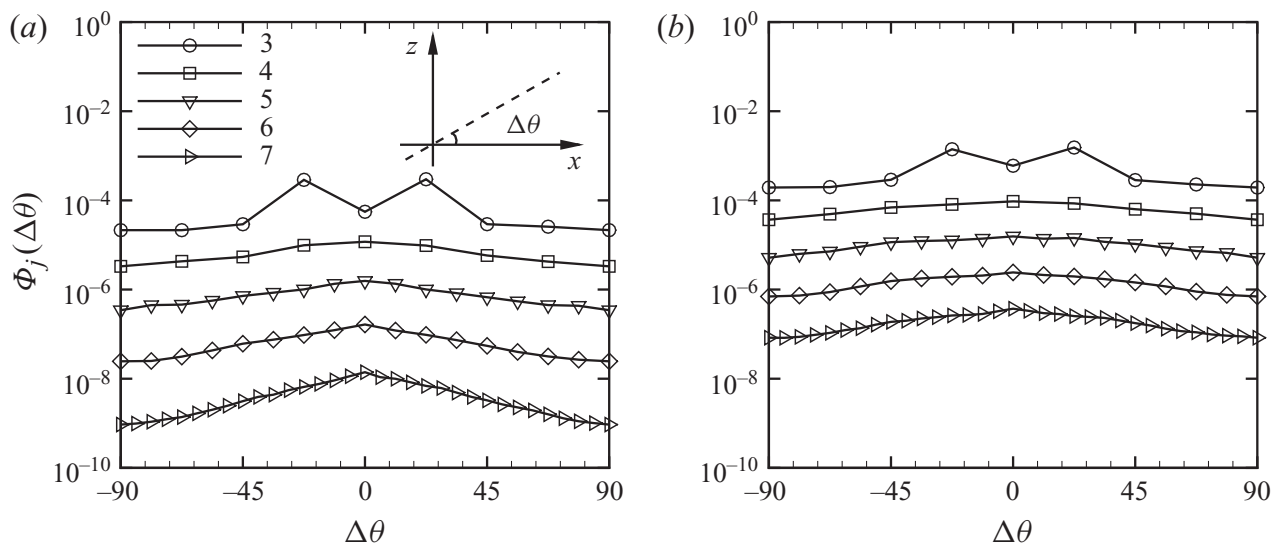

Figure 10. Angular spectra of Lagrangian fields at different scales on the $x-z$ plane in turbulent channel flow for $\operatorname{Re}_{\tau}=395$ (run S2). The deviation angle $\Delta \theta$ is illustrated in the upper-right of $(a)$. (a) $t_{c}=1$ and $(b) t_{c}=4$.

(a)

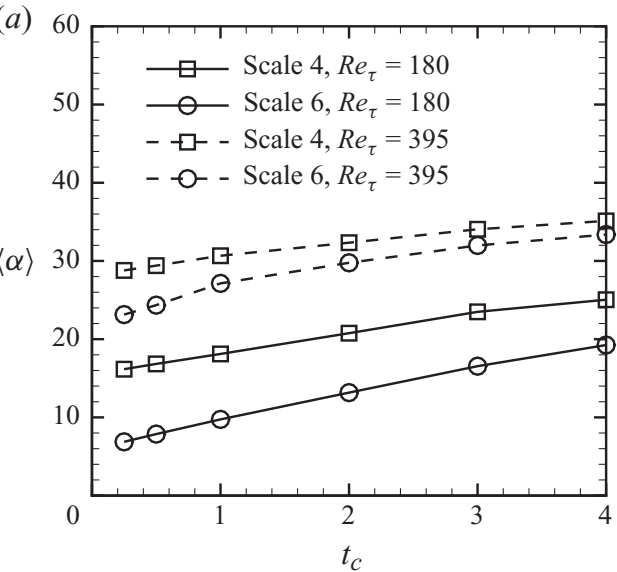

(b)

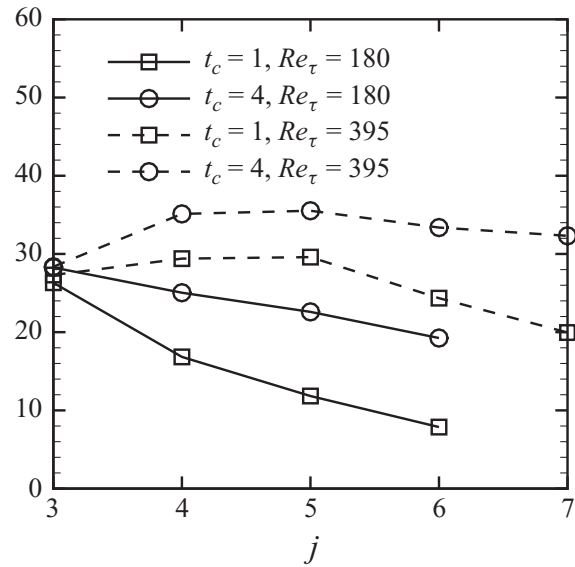

FIGURE 11. Evolution of the averaged inclination angle (degrees) of Lagrangian structures on the $x-z$ plane in turbulent channel flow for $R e_{\tau}=180$ (run S1) and $R e_{\tau}=395$ (run S2). (a) Different times and $(b)$ different scales.

geometries of Lagrangian structures, discussed by Yang et al. (2010) for forced isotropic turbulence, may also exist in turbulent channel flow.

\subsection{Geometry of Lagrangian structures on the $x-y$ (streamwise and spanwise) plane}

Quasi-streamwise vortices within candidate hairpin- or $\Lambda$-vortices show, in addition to the wall inclination, another geometric feature that can be characterized by the sweep angle $\beta$ between the elongated structure and the $x$-direction on the $x-y$ plane at $z=z_{p}$ (see figure 1). In terms of the variables in the multi-scale and multi-directional method as shown in figure 4 , we have $\phi\left(x, y, z=z_{p}\right) \leftrightarrow \varphi, x \leftrightarrow x_{1}, y \leftrightarrow x_{2}, \beta^{+} \leftrightarrow\langle\Delta \theta\rangle^{+}$and $\beta^{-} \leftrightarrow\langle\Delta \theta\rangle^{-}$. Although an individual structure may have asymmetrical legs in lowReynolds-number flows (Robinson 1991), we find that the difference of $\left\langle\beta^{+}\right\rangle$and $\left\langle\beta^{-}\right\rangle$ is typically less than $10 \%$ for each plane. Hence, we assume statistically symmetry over structures in the channel and define the averaged sweep angle $\langle\beta\rangle=\left(\left\langle\beta^{+}\right\rangle+\left\langle\beta^{-}\right\rangle\right) / 2$. 
(a)

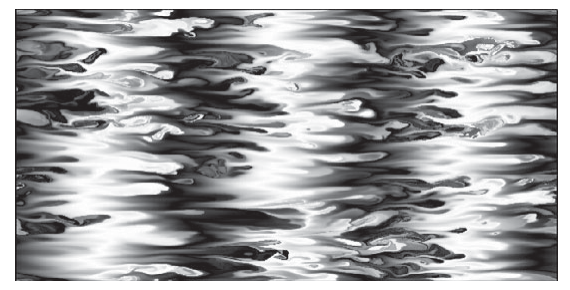

(c)

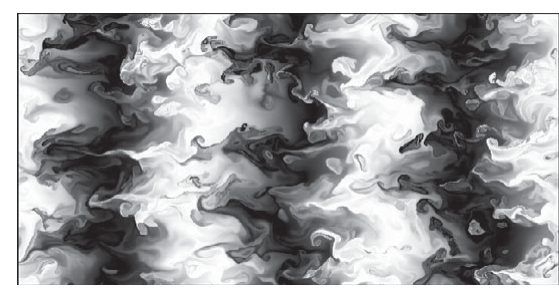

(b)

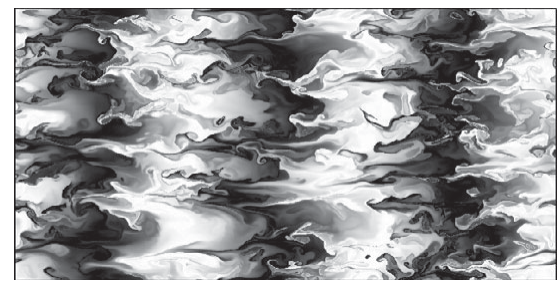

(d)

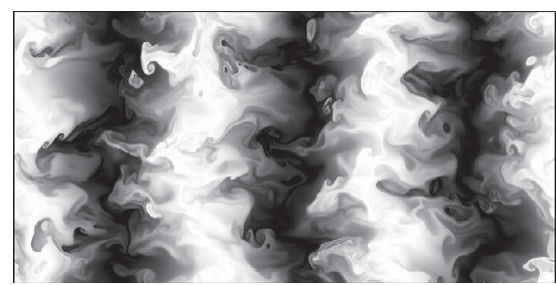

FIGURE 12. Lagrangian structures on the $x-y$ plane $(0 \leqslant x \leqslant 2 \pi, 0 \leqslant y \leqslant \pi)$ at $t_{c}=2$ in turbulent channel flow for $R e_{\tau}=395$ (run S2). (a) $z^{+}=5$, viscous sublayer, $(b) z^{+}=30$, buffer layer, (c) $z^{+}=60$, logarithm law region and $(d) z^{+}=120$, outer layer.

For investigating the evolutionary geometry of Lagrangian structures on the $x-y$ plane, the initial Lagrangian field is chosen as $\phi_{0}=\sin 3 x$. Starting from streaks with characteristic scale $\delta$ normal to the streamwise direction, at a typical time $t_{c}=2$, figure 12 shows different geometries of Lagrangian structures on the $x-y$ plane in different regions as characterized by Pope (2000). In the viscous sublayer, spiky-like, long narrow streaks in the $x$-direction are observed, which may be generated by the shear motion from high- and low-speed alternating streamwise velocity streaks very close to the wall. We can see that the structures are still relatively smooth, which shows that turbulent fluctuations are relatively small in the viscous sublayer (Kline et al. 1967). In the buffer layer and the logarithmic region, we find obvious predominant $\Lambda$-like structures, while structures in the buffer layer with active turbulent energy production that exhibit the 'bursting process' (Kline et al. 1967) appear to be more chaotic than those in the logarithmic region. In comparison, structures in the outer layer are much smoother and less perturbed than those in the inner layer.

The averaged sweep angle $\langle\beta\rangle$ for two Reynolds numbers is plotted in figure 13 . We find that, in general, $\langle\beta\rangle$ at all scales increases with wall distance $z^{+}$as revealed in figure 12. Using hot-wire measurements, Ong \& Wallace (1998) found that the sweep angle of vorticity vectors increased with distance from the wall, which is consistent with our results for Lagrangian structures. The averaged sweep angle $\langle\beta\rangle$ of structures at several scales increases rapidly with increasing $z^{+}$in the buffer layer with $5 \leqslant y^{+} \leqslant 30$, and in the logarithmic law region with $y^{+} \geqslant 30$ and $y / \delta \leqslant 0.3$, and grows slower in the outer layer with $y^{+}>50$. This might be related to active turbulent production occurring in this region, with less activity in the outer layer (e.g. Adrian 2007). On the other hand, the growth of $\langle\beta\rangle$ at the intermediate scale around $50 \delta_{v}$ is slower than those at smaller scales. The corresponding visual inspection is also shown in figure 14.

The temporal evolution of Lagrangian structures on the $x-y$ plane in the buffer layer of turbulent channel flow at $R e_{\tau}=395$ and at different times is shown in figure 15 . Starting with smooth tube-like shapes, we can see the $\Lambda$-shape structures induced 
(a)

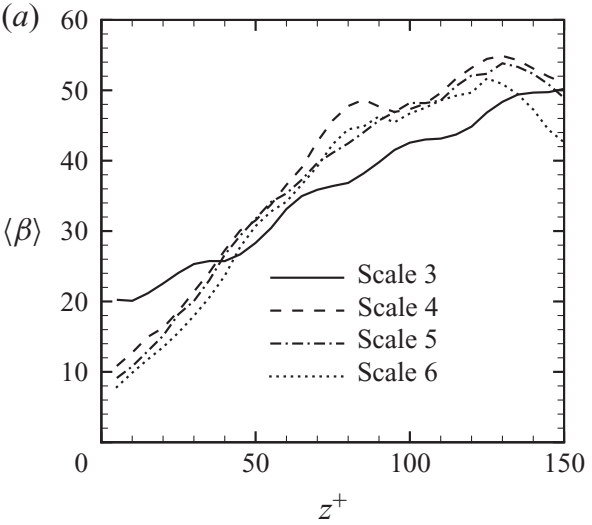

(b)

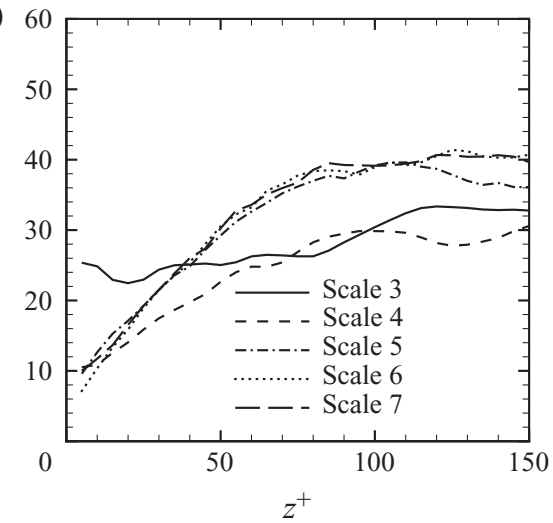

FIGURE 13. Averaged sweep angle (degrees) of Lagrangian structures on the $x-y$ plane at $t_{c}=2$ in turbulent channel flow for $R e_{\tau}=180$ (run S1) and $R e_{\tau}=395$ (run S2). (a) $R e_{\tau}=180$ and $(b) R e_{\tau}=395$.

(a)

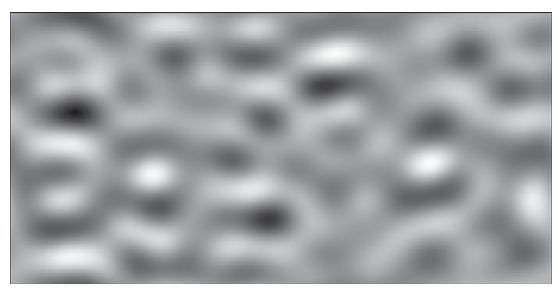

(c)

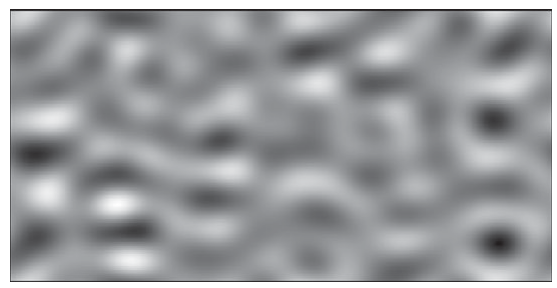

(e)

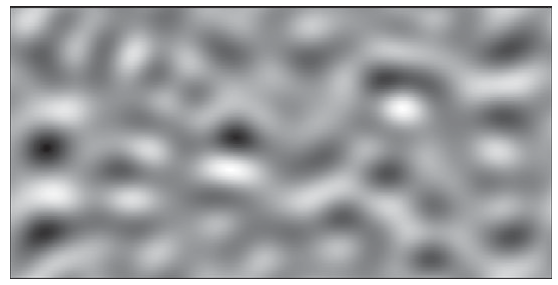

(b)

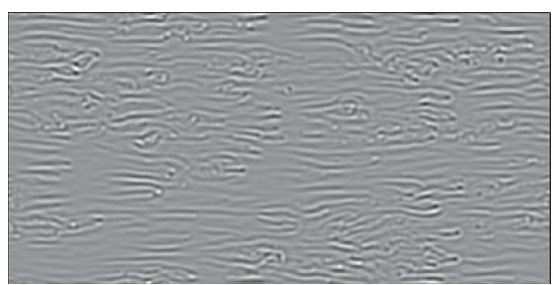

(d)

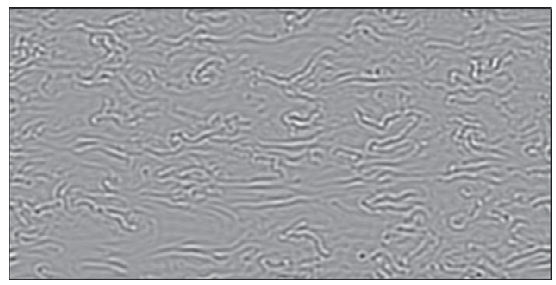

$(f)$

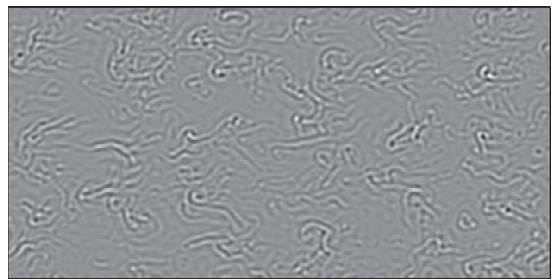

FIGURE 14. Lagrangian structures on the $x-y$ plane $(0 \leqslant x \leqslant 2 \pi, 0 \leqslant y \leqslant \pi)$ at $t_{c}=2$ and different scales in turbulent channel flow for $R e_{\tau}=395$ (run S2). (a) Scale $3, z^{+}=5$, viscous sublayer, $(b)$ scale $6, z^{+}=5$, viscous sublayer, $(c)$ scale $3, z^{+}=30$, buffer layer, $(d)$ scale 6 , $z^{+}=30$, buffer layer, $(e)$ scale $3, z^{+}=30$, logarithm law region and $(f)$ scale $6, z^{+}=60$, logarithm law region.

by the near-wall, low-speed streaks at early time followed by intense fluctuations at later time, which shows active energy production and transfer in the buffer layer. In figure 16, the corresponding temporal evolution of $\langle\beta\rangle$ in the channel flow at 
(a)

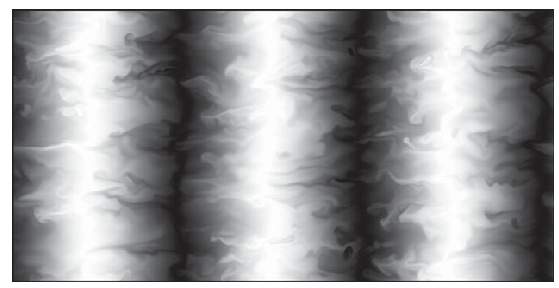

(c)

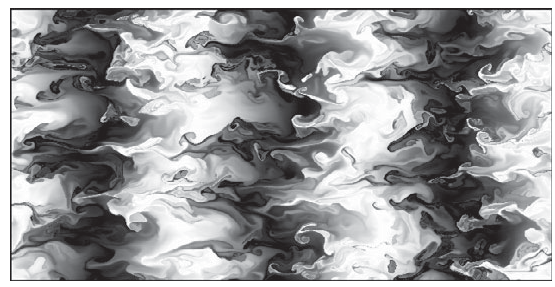

(b)

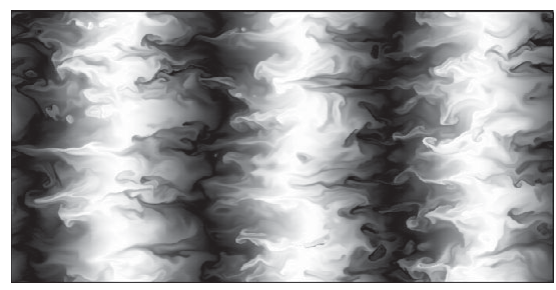

(d)

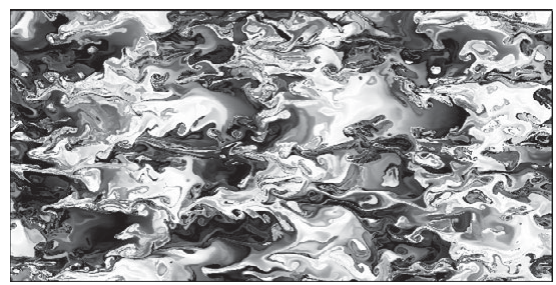

FIGURE 15. Evolution of Lagrangian structures at $z^{+}=45$ on the $x-y$ plane $(0 \leqslant x \leqslant 2 \pi$, $0 \leqslant y \leqslant \pi$ ) in turbulent channel flow for $\operatorname{Re}_{\tau}=395$ (run S2). (a) $t_{c}=0.5,(b) t_{c}=1,(c) t_{c}=2$ and (d) $t_{c}=4$.
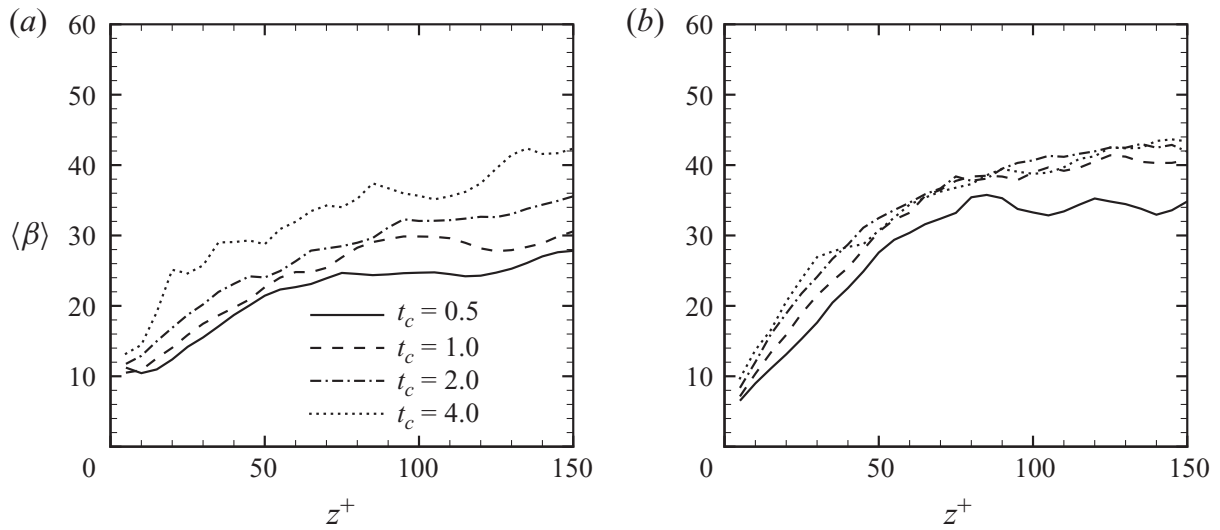

FiguRE 16. Evolution of the averaged sweep angle (degrees) of Lagrangian structures on the $x-y$ plane in the turbulent channel flow for $R e_{\tau}=395$ (run S2). (a) Scale $=4$ and (b) scale $=6$.

$R e_{\tau}=395$ shows that the averaged sweep angle of Lagrangian structures grows with time. Combined with the results shown in $\S 4.1$, we show that, starting from initial smooth large-scale blob- or tube-like shapes, Lagrangian structures may breakdown and evolve into possible hairpin- or $\Lambda$-like shapes at intermediate and small scales.

\section{Eulerian structures in turbulent channel flow}

\subsection{Geometry of Eulerian structures on the $x-z$ plane or $x-y$ plane}

We extract Eulerian structures as iso-contours of the instantaneous swirling strength field $\lambda_{c i}$ in turbulent channel flow. Here, $\lambda_{c i}$ is the imaginary part of the complex eigenvalue of the velocity gradient tensor $\nabla \boldsymbol{u}$ (see Zhou et al. 1999), which is often used to identify vortical structures in wall turbulence. Similarities and differences between 
(a)

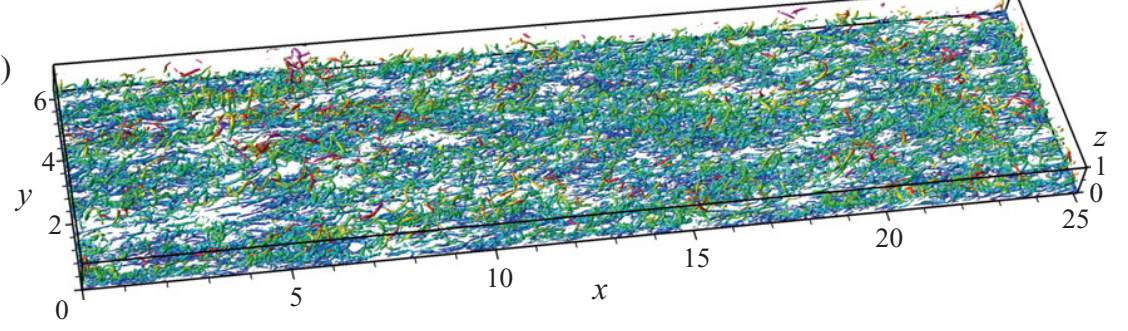

(b)

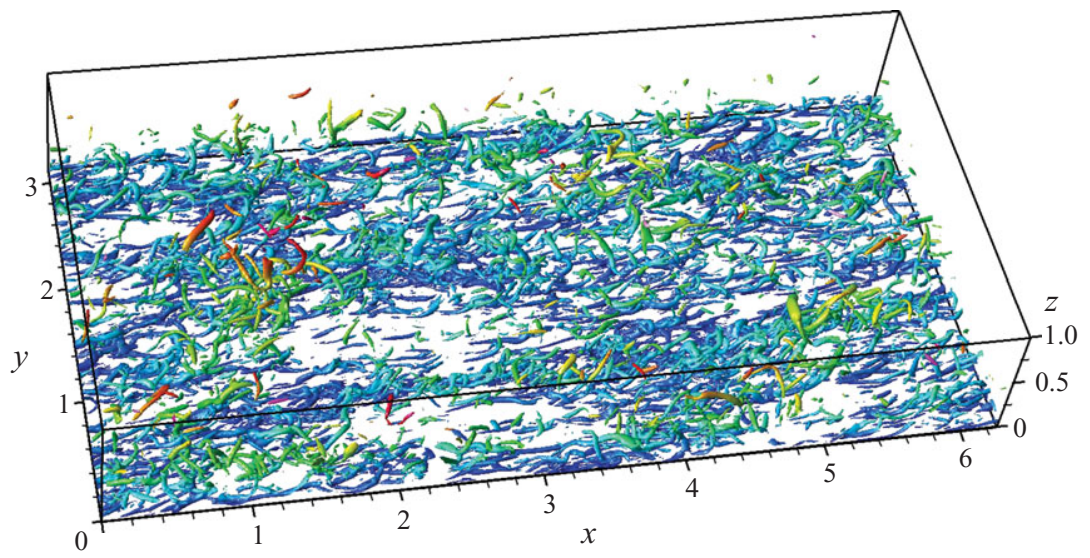

FIGURE 17. (Colour online available at journals.cambridge.org/FLM) Snapshots of Eulerian structures (iso-surfaces at $\lambda_{c i} / \lambda_{c i, \max }=0.1$ ) in turbulent channel flows. The colour on the surfaces is coded by $z$. (a) $R e_{\tau}=395$ (run L2) and (b) $R e_{\tau}=590$ (run S3).

the swirling strength and other vortex identification criteria were discussed in Chakraborty, Balachandar \& Adrian (2005). Remarkably similar looking vortical structures, obtained using their criteria with the given proposed usage of threshold, were observed in a canonical turbulent flow example. The visualization of iso-surfaces at $\lambda_{c i} / \lambda_{c i, \max }=0.1$ in turbulent channel flows is shown in figure 17 . Numerous tube-like structures extended in the streamwise direction and inclined to the wall can be seen. Some appear to form complete or incomplete hairpin- or $\Lambda$-like structures. Similar observations were also made by Adrian \& Liu (2002) and Ganapathisubramani et al. (2006).

After interpolating $\lambda_{c i}$ on a uniform grid with resolution $2 N_{x} \times N_{y} \times N_{z}$, the multiscale and multi-directional methodology described in $\S 3$ is applied on a sequence of $\lambda_{c i}$-fields on the $x-y$ or $x-z$ planes. In this subsection, we will use Eulerian velocity fields from runs L1, L2, S3 and S4 (see table 1) for four Reynolds numbers. The scale decomposition of Eulerian structures on each plane is obtained by (3.15), where the scalar field $\varphi=\lambda_{c i}\left(x, y=y_{p}, z\right)$. For example, the scale decomposition of the $\lambda_{c i}$-field on the $x-z$ plane for $R e_{\tau}=590$ is shown in figure 18. Clearly evident are decreasing characteristic length scales with increasing scale indices, as quantified in table 2.

The $\lambda_{c i}$-field on the $x-z$ plane with non-periodic boundaries is mirror-extended as (3.22) for the purpose of further analysis. The averaged inclination angle $\langle\alpha\rangle$ of Eulerian structures on the $x-z$ plane at different scales and Reynolds numbers is shown in figure $19(a)$. Additional averaging on $\langle\alpha\rangle$ was taken over $50 x-z$ planes at $y=y_{p}$ uniformly distributed between $y=0$ and $y=L_{y}$ from five independent realizations of the DNS. We can see that the averaged inclination angles, $\langle\alpha\rangle$, of 
(a)

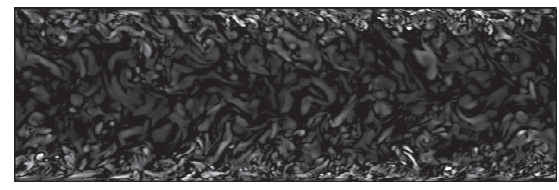

(c)

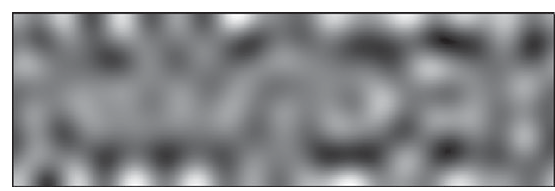

(e)

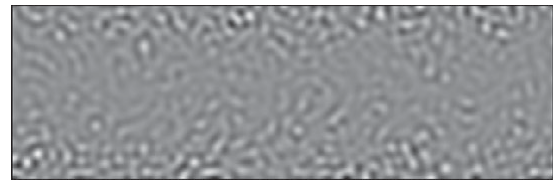

(b)

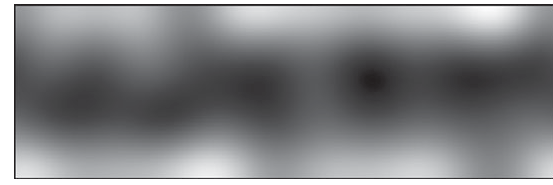

(d)

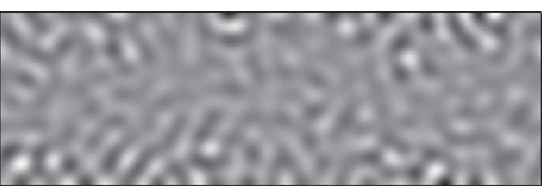

$(f)$

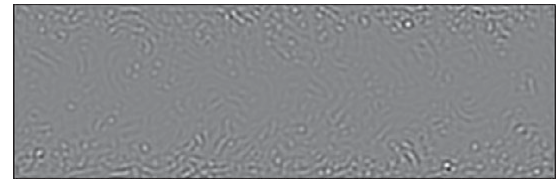

FigurE 18. Scale decomposition of Eulerian structures on the $x-z$ plane $(0 \leqslant x \leqslant 2 \pi, 0 \leqslant z \leqslant 2)$ in turbulent channel flow for $R e_{\tau}=590$ (run S3). (a) Original, (b) scale 2, (c) scale 3, $(d)$ scale 4 , (e) scale 5 and $(f)$ scale 6.

(a)

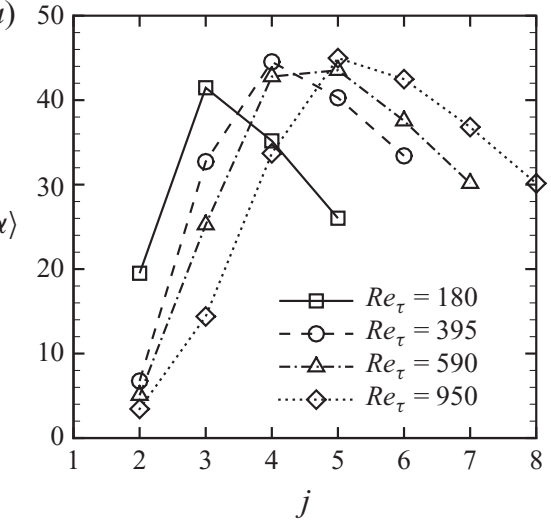

(b)

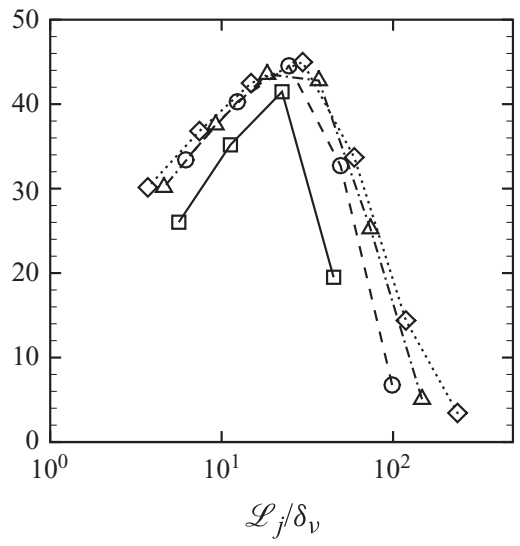

FIGURE 19. Averaged inclination angle (degrees) of Eulerian structures on the $x-z$ plane. $(a)$ Scaled by scale indices and $(b)$ scaled by viscous length scales.

large-scale Eulerian structures are small, while structures at intermediate scales have maximum $\langle\alpha\rangle$ in the range $40^{\circ}-45^{\circ}$ while small-scale structures have moderate $\langle\alpha\rangle$ around $30^{\circ}$. In figure $19(b)$, the $\langle\alpha\rangle$ profiles, in terms of the viscous scale $\delta_{v}$, appear to collapse for all four Reynolds numbers, in a way similar to inner-scaling for turbulent velocity or vorticity profiles (e.g. Moser et al. 1999). The maximum $\langle\alpha\rangle$ corresponds to the scale around $20 \delta_{v}$. Combined with the visualizations in figure 18 , we conclude that the quasi-streamwise vortices represented by iso-surfaces of $\lambda_{c i}$ exhibit an inclined geometry with intermediate scale $20 \delta_{v},\langle\alpha\rangle \approx 45^{\circ}$ and with 'curved legs' leading to scale $5 \delta_{v}$ and $\langle\alpha\rangle \approx 30^{\circ}$ near the wall. 
(a)

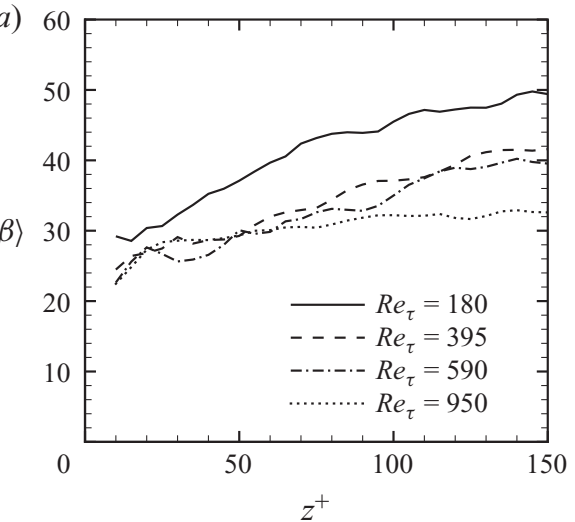

(b)

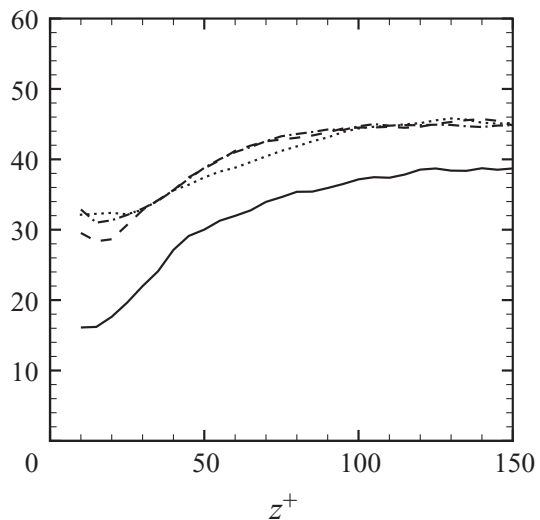

Figure 20. Averaged sweep angle (degrees) of Eulerian structures on the $x-y$ plane at different $z^{+}$. (a) Scale 3 and $(b)$ scale 5 .

After averaging over results from five realizations of the DNS, the averaged sweep angle $\langle\beta\rangle$ of Eulerian structures on the $x-z$ plane as a function of the wall distance $z^{+}$ for four Reynolds numbers is also shown in figure 20. This resembles the equivalent results for Lagrangian structures shown in $\S 4$. The rapid change of the sweep angle in the near-wall region implies high curvature of three-dimensional small-scale Eulerian structures (e.g. Adrian 2007).

\subsection{Statistical evidence of structure packets}

Recent DNS and PIV studies have provided evidence that near-wall vortices may group together to form large-scale packets (Zhou et al. 1999; Christensen \& Adrian 2001; Adrian \& Liu 2002). We observed that similar long and narrow structures appear to be organized by a number of individual small-scale structures in figure 17 . To investigate this issue by quantified statistics, we use the cross-correlation coefficients between the $\lambda_{c i}$ fields on the $x-z$ plane at scale $i$ and scale $j$ :

$$
\gamma_{i j}=\frac{\left\langle\left(\left|\lambda_{c i}^{(i)}(\boldsymbol{x})\right|-\left\langle\left|\lambda_{c i}^{(i)}(\boldsymbol{x})\right|\right\rangle\right)\left(\left|\lambda_{c i}^{(j)}(\boldsymbol{x})\right|-\left\langle\left|\lambda_{c i}^{(j)}(\boldsymbol{x})\right|\right\rangle\right)\right\rangle}{\sigma^{(i)} \sigma^{(j)}},
$$

where $i<j$ and the standard deviation of each scalar field is

$$
\sigma^{(j)}=\sqrt{\left\langle\left(\left|\lambda_{c i}^{(j)}(\boldsymbol{x})\right|-\left\langle\left|\lambda_{c i}^{(j)}(\boldsymbol{x})\right|\right\rangle\right)^{2}\right\rangle}
$$

In the multi-scale geometric methodology, the structures are represented by fluctuations of $\lambda_{c i}^{(j)}$. Thus, high $\gamma_{i j}$ means possible coincidence of structures at scales $i$ and $j$ appearing within the same region. In figure $21, \gamma_{i j}$ is computed by (5.1) on the $x-z$ plane in channel flows for four Reynolds numbers. We can see noticeable cross-correlations between large-scale structures with the characteristic height $0.25 \delta$ and small-scale structures with the length around $5 \delta_{v}$ in both long channel (runs L1, L2 and L3) and short channel (runs S2, S3 and S4), which implies that candidate structure packets may exist in the near-wall region of channel flows at a range of Reynolds numbers. This feature can be observed from visualizations of the $x$ $z$ plane-cuts in figure 22. The small-scale structures presented by the fluctuations of the background shading of $\lambda_{c i}^{(5)}$ for $R e_{\tau}=180$ or $\lambda_{c i}^{(6)}$ for $R e_{\tau}=395$ are grouped in large-scale packets presented by dashed contour lines of $\lambda_{c i}^{(2)}$. 
(a)

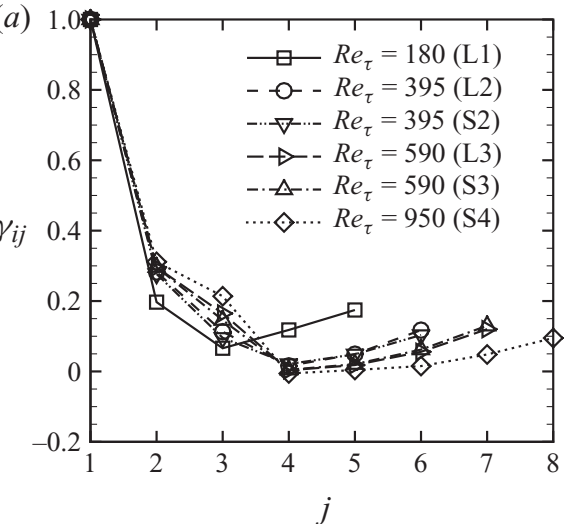

(b)

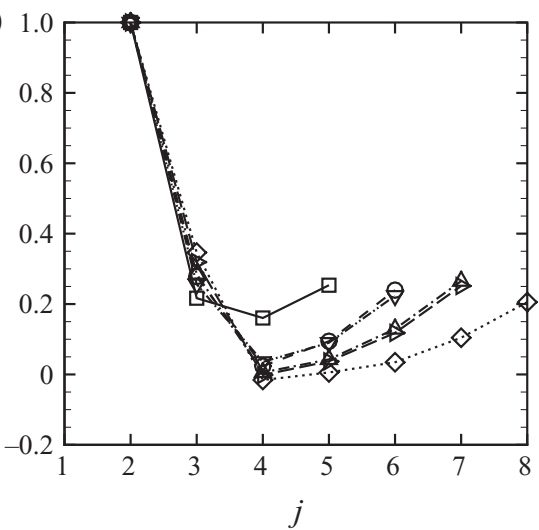

FIGURE 21. Cross-correlation coefficients between Eulerian structures at scale $i$ and scale $j$ from different runs. (a) $i=1$ and $(b) i=2$.

(a)

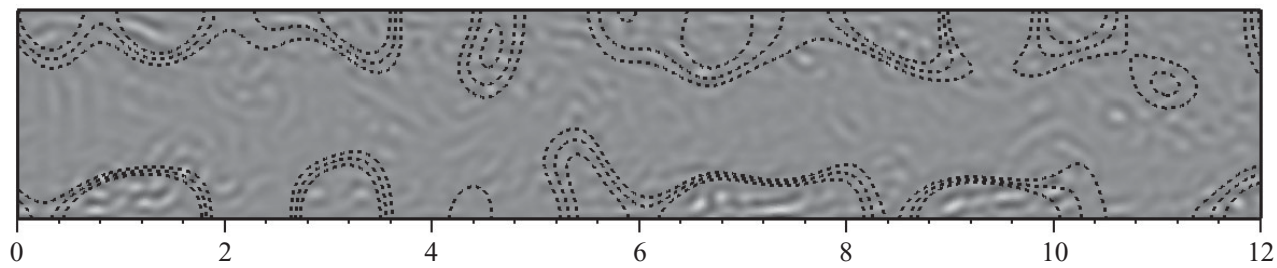

(b)

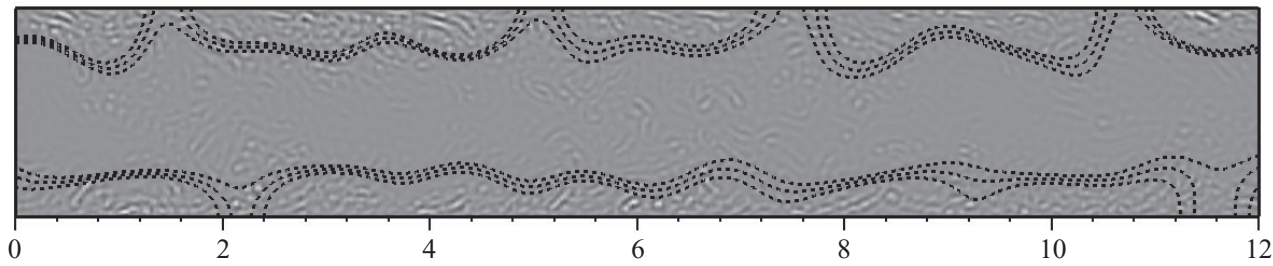

FiguRE 22. Interactions between Eulerian large-scale structures at scale $i$ (contour lines: $\lambda_{c i}^{(i)} / \lambda_{c i, \max }^{(i)}=0.15,0.2,0.25$ ) and small-scale structures at scale $j$ (background shading) on the $x-y$ plane in a long channel. (a) $R e_{\tau}=180$ (run L1), $i=2, j=5,(b) R e_{\tau}=395$ (run L2), $i=2$ and $j=6$.

Additionally, in long channel flows with $L_{x} \approx 50 \delta$ at $R e_{\tau}=180$ and $R e_{\tau}=395$ (runs L1 and L2), as shown in figure 23, large-scale structures in the higher-Reynoldsnumber flow are much longer in the streamwise direction than those in the lowerReynolds-number flow. A characteristic length of structures at scale $j$ in the $x$ direction can be defined as the integral length scale

$$
L_{x}^{(j)}=\int_{0}^{L_{x} / 2} R_{x}^{(j)}\left(r_{x}\right) \mathrm{d} r_{x}
$$

where the correlation in the streamwise direction is

$$
R_{x}^{(j)}\left(r_{x}\right)=\frac{\left\langle\left(\lambda_{c i}^{(j)}(\boldsymbol{x})-\left\langle\lambda_{c i}^{(j)}(\boldsymbol{x})\right\rangle\right)\left(\lambda_{c i}^{(j)}\left(x+r_{x}, y=y_{p}, z\right)-\left\langle\lambda_{c i}^{(j)}(\boldsymbol{x})\right\rangle\right)\right\rangle}{\left\langle\left(\lambda_{c i}^{(j)}(\boldsymbol{x})-\left\langle\lambda_{c i}^{(j)}(\boldsymbol{x})\right\rangle\right)^{2}\right\rangle} .
$$


(a)

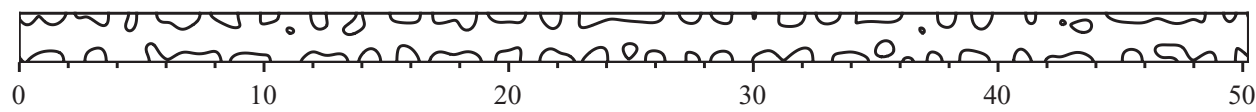

(b)

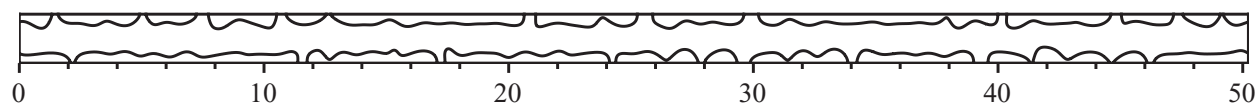

FIGURE 23. Eulerian structures (contour lines: $\lambda_{c i}^{(j)} / \lambda_{c i \text { max }}^{(j)}=0.2$ ) at scale 2 on the $x-z$ plane $\left(0 \leqslant x \leqslant 16 \pi, 0 \leqslant z \leqslant 2\right.$ ) in long channel flows. (a) $R e_{\tau}=180$ (run L1) and (b) $R e_{\tau}=395$ (run L2).

We find that the maximum $L_{x}^{(j)}$ are obtained when $j=2$ in both Reynolds-number flows, while $L_{x}^{(2)} \approx 4 \delta$ in the flow at $R e_{\tau}=180$ and $L_{x}^{(2)} \approx 14 \delta$ in the flow at $R e_{\tau}=395$, which might imply that it is easier to form very long structure packets in higherReynolds-number flows than in low-Reynolds-number flows. The latter result is reminiscent of that obtained from the streamwise spectra in the experiments of turbulent pipe flow over a range of Reynolds numbers (e.g. Kim \& Adrian 1999; Guala et al. 2006).

\subsection{Comparisons of Lagrangian and Eulerian structures}

Eulerian structures show many geometric features that are qualitatively similar to those of Lagrangian structures. This similarity also supports the existence of quasi-streamwise vortices in turbulent channel flow. There are nonetheless notable differences in our analysis of these two classes of fields. Some of these, for example the averaged inclination and sweep angles of structures, may result from the Lagrangian/Eulerian identification methods themselves, while other may have a more physical basis. Lagrangian structures tend to be stretched by the persistent shear motions thereby generating elongated, curved structures partially attached to the wall as illustrated in figure 8. In Eulerian fields, the swirling-strength field appears to identify vortex cores in the logarithmic region with relatively short geometry as shown in figure 18. This can be expected to produce smaller averaged inclination angles for Lagrangian objects than those seen for Eulerian structures at intermediate scales.

Another notable difference is that, in contrast to the observation of structure packets in terms of correlations for Eulerian structures (figure 21), we do not presently find substantial cross-correlations between large- and small-scale components of Lagrangian objects. This could be a consequence of the limited time over which we are able to track the latter, which may not be sufficiently long for inter-scale Lagrangian correlations to develop. An alternative is that the Eulerian correlations are in fact a consequence of the collective vortex dynamics of near-wall flows. Presently we do not have a suitable method available for the long-time tracking of a Lagrangian field that is unambiguously related to identifiable vortex structures; see further discussion below.

One clear distinction between Lagrangian and Eulerian fields is that the former are the result of evolution from essentially arbitrary initial conditions, while the latter follow from iso-surfaces of scalar quantities chosen at least in part based on vorticity physics. Yang et al. (2010) found Lagrangian structures in isotropic turbulence that were broadly independent of initial fields with blob-like, tube-like or sheet-like isosurfaces. Presently we find a similar trend for turbulent channel flow. 
A connection between Lagrangian and Eulerian field geometries could perhaps be educed by choice of initial conditions for the Lagrangian simulation that correspond to a physically interesting Eulerian field. In order to investigate Lagrangian mechanisms and vortex dynamics in idealized flows with Taylor-Green or Kida-Pelz initial conditions, Yang \& Pullin (2010) introduced the vortex-surface field $\phi=\phi_{v}$ satisfying $\lambda_{\omega}=0$, where

$$
\lambda_{\omega}=\frac{\omega \cdot \nabla \phi}{|\omega||\nabla \phi|} .
$$

In strictly inviscid flow, the Helmholtz vorticity theorems show that $\phi(x, t=0)$ satisfying $\lambda_{\omega}=0$ at $t=0$ will do so for $t>0$. This fails for viscous flow. Further, for a given $\omega$ field, there are open existence and uniqueness questions concerning the determination of $\phi$ satisfying $\lambda_{\omega}=0$. A surrogate field may nevertheless be useful. For the present turbulent channel flows, we find that the choice $\phi=\lambda_{c i}$ or $\phi=|\omega|$ obtained from the instantaneous Eulerian velocity field gives $\left\langle\left|\lambda_{\omega}\right|\right\rangle \approx 0.5$. While neither of these is then close to a vortex-surface field, they may nevertheless provide interesting initial Lagrangian fields. Hence, in addition to the initial Lagrangian fields described in $\S \S 4.1$ and 4.2, we also performed simulations of Lagrangian structures evolving from initial conditions defined by the three-dimensional filtered $\lambda_{c i}$-field at scale 2 . This produced Lagrangian fields at a later time similar to those presented in $\$ \S 4.1$ and 4.2.

\section{Conclusions}

We have developed a general multi-scale, multi-directional methodology based on the mirror-extended curvelet transform to investigate the geometry of Lagrangian and Eulerian structures, extracted respectively from a time sequence of the Lagrangian fields and from the instantaneous swirling-strength field in turbulent channel flow, for low and moderate Reynolds numbers. This is used to quantify the statistical geometry, including the averaged inclination and sweep angles, of both classes of structures over a range of scales varying from the half-height of the channel to several viscous length scales. Quasi-streamwise Lagrangian and Eulerian vortical structures were detected in the near-wall region and their geometries quantified. These comprise inclined objects, the averaged inclination angle of which is $35^{\circ}-45^{\circ}$ principally within the logarithmic region. The averaged sweep angle is $30^{\circ}-40^{\circ}$ and the characteristic scale is $20 \delta_{v}$. 'Curved legs' are found in the viscous sublayer and buffer layer, for which the averaged inclination angle is $20^{\circ}-30^{\circ}$, the averaged sweep angle is $15^{\circ}-30^{\circ}$ and the scale is $5 \delta_{v}-10 \delta_{v}$. The sweep angle of both structures increases rapidly in the buffer and logarithmic regions and grows mildly in the outer layer. The temporal evolution of Lagrangian structures shows increasing inclination and sweep angles with time. The increasing magnitude in terms of both angles varies from $10^{\circ}$ to $20^{\circ}$ within the typical 'cyclic' period from $t_{c}=0.5$ to $t_{c}=4$. This may quantify the lifting process of quasi-streamwise vortices and the conceptional ejection-sweep-burst-inrush scenario. Both structures have slightly different geometries in flows for different Reynolds numbers. Although the averaged geometrical features of these objects are consistent with the expected signatures of conceptual structures previously characterized as hairpin or $\Lambda$-vortices, we remark that the current methodology cannot distinguish between hairpin-like structures composed of two connected tubes and inclined tubelike structures that are not connected to each other via vortex lines.

Evidence for the existence of large-scale, Eulerian structure packets, comprising collections of individual small-scale geometrical objects, was obtained by finite 
cross-correlations between large- and small-scale Eulerian structures. The large-scale packets are located within the near-wall region with the typical height $0.25 \delta$ and may extend over $10 \delta$ in the streamwise direction in moderate-Reynolds-number, long channel flows.

The current methodology is based on a sequence of plane-cuts normal or parallel to the streamwise direction in channel flows and so may also be suitable and convenient for analysing experimental PIV data. The extension to fully three-dimensional data could be achieved using the three-dimensional curvelet transform (Ying, Demanet \& Candès 2005). The fast discrete curvelet transform algorithm with circular frequency window functions may be required for this. In addition, combined with the mirrorextension, the multi-scale geometric analysis (Bermejo-Moreno \& Pullin 2008) using the fast three-dimensional curvelet transform can in principle be applied to detect coherent structures in wall turbulence in order to study alternative non-local geometry signatures based on principal curvatures. This could provide a means of testing an assumed structure in simplified models for wall turbulence (e.g. Perry \& Chong 1982; Perry et al. 1986), structure-based subgrid models for the LES of near-wall channel (Chung \& Pullin 2009) or boundary layer flows and the possible sparse representation of wall turbulence with the curvelet-based extraction method.

While the data analysed in this study are from low- to moderate-Reynolds-number channel flows, the present multi-scale and multi-directional methodology can easily be applied to high-Reynolds-number data (e.g. Marusic et al. 2010) for turbulent channel or boundary layer flows to explore more geometric features such as the superstructures in the outer layer (e.g. Hutchins \& Marusic 2007) and the structural evolution in the turbulent transition (e.g. Wu \& Moin 2009). Moreover, since high-Reynolds-number wall turbulence exhibits scale separation, it would be interesting to investigate various inter-scale interactions such as the large-scale modulation of small-scale motions and Reynolds stresses using the curvelet multi-scale decomposition with quadrant analysis (e.g. Wallace, Brodkey \& Eckelman 1972).

The authors are grateful to P. Koumoutsakos for providing generous access on the Brutus cluster at the ETH Zurich. The authors thank I. Bermejo-Moreno and D. Chung for helpful comments. This work has been supported in part by the National Science Foundation under grant DMS-1016111.

\section{REFERENCES}

Adrian, R. J. 2007 Hairpin vortex organization in wall turbulence. Phys. Fluids 19, 041301.

Adrian, R. J. \& LiU, Z. C. 2002 Observation of vortex packets in direct numerical simulation of fully turbulent channel flow. J. Vis. 5, 9-19.

del Álamo, J. C., JimÉnez, J., Zandonade, P. \& Moser, R. D. 2004 Scaling of the energy spectra of turbulent channels. J. Fluid Mech. 500, 135-144.

BAmberger, R. H. \& SMith, M. J. T. 1992 A filter bank for the directional decomposition of images: theory and design. IEEE Trans. Signal Process. 40, 882-893.

BANDYOPADHYAY, P. 1980 Large structure with a characteristic upstream interface in turbulent boundary-layers. Phys. Fluids 23, 2326-2327.

Bermejo-Moreno, I. \& Pullin, D. I. 2008 On the non-local geometry of turbulence. J. Fluid Mech. 603, 101-135.

Candès, E., Demanet, L., Donoho, D. \& Ying, L. 2006 Fast discrete curvelet transforms. Multiscale Model. Simul. 5, 861-899.

Chakraborty, P., Balachandar, S. \& Adrian, R. J. 2005 On the relationships between local vortex identification schemes. J. Fluid Mech. 535, 189-214. 
Chong, M. S., Perry, A. E. \& Cantwell, B. J. 1990 A general classification of three-dimensional flow fields. Phys. Fluids A 2, 765-777.

Christensen, K. T. \& Adrian, R. J. 2001 Statistical evidence of hairpin vortex packets in wall turbulence. J. Fluid Mech. 431, 433-443.

Chung, D. \& Pullin, D. I. 2009 Large-eddy simulation and wall modelling of turbulent channel flow. J. Fluid Mech. 631, 281-309.

Demanet, L. \& Ying, L. 2007 Curvelets and wave atoms for mirror-extended images. In Proceedings of the Society of Photo-optical Instrumentation Engineers (SPIE), vol. 6701, p. 67010J. SPIE.

FALCO, R. E. 1977 Coherent motions in outer region of turbulent boundary-layers. Phys. Fluids 20, S124-S132.

Ganapathisubramani, B., Longmire, E. K. \& Marusic, I. 2006 Experimental investigation of vortex properties in a turbulent boundary layer. Phys. Fluids 18, 055105.

Green, M. A., Rowley, C. W. \& Haller, G. 2007 Detection of Lagrangian coherent structures in three-dimensional turbulence. J. Fluid Mech. 572, 111-120.

Guala, M., Hommema, S. E. \& Adrian, R. J. 2006 Large-scale and very-large-scale motions in turbulent pipe flow. J. Fluid Mech. 554, 521-542.

HALLER, G. 2001 Distinguished material surfaces and coherent structures in three-dimensional fluid flows. Physica D 149, 248-277.

Head, M. R. \& Bandyopadhyay, P. 1981 New aspects of turbulent boundary-layer structure. J. Fluid Mech. 107, 297-338.

Hinze, J. O. 1975 Turbulence, 2nd edn. McGrow-Hill.

Honkan, A. \& ANDreopoulos, Y. 1997 Vorticity, strain-rate and dissipation characteristics in the near-wall region of turbulent boundary layers. J. Fluid Mech. 350, 29-96.

Hunt, J. C. R., Wray, A. A. \& Moin, P. 1988 Eddies, stream, and convergence zones in turbulent flows. Center for Turbulence Research Report CTR-S88, pp. 193-208.

Hutchins, N. \& Marusic, I. 2007 Evidence of very long meandering features in the logarithmic region of turbulent boundary layers. J. Fluid Mech. 579, 1-28.

JeOng, J. \& Hussain, F. 1995 On the identification of a vortex. J. Fluid Mech. 285, 69-94.

JimÉnez, J., Del Álamo, J. C. \& FloRes, O. 2004 The large-scale dynamics of near-wall turbulence. J. Fluid Mech. 505, 179-199.

Kim, J., Moin, P. \& Moser, R. 1987 Turbulence statistics in fully-developed channel flow at low Reynolds-number. J. Fluid Mech. 177, 133-166.

Kim, K. C. \& Adrian, R. J. 1999 Very large-scale motion in the outer layer. Phys. Fluids 11, 417-422.

Kline, S. J., Reynolds, W. C., Schraub, F. A. \& Runstadl, P. W. 1967 Structure of turbulent boundary layers. J. Fluid Mech. 30, 741-773.

Liu, Z. C., Landreth, C. C., Adrian, R. J. \& Hanratty, T. J. 1991 High-resolution measurement of turbulent structure in a channel with particle image velocimetry. Exp. Fluids 10, 301-312.

Ma, J., Hussaini, M. Y., Vasilyev, O. V. \& Le Dimet, F.-X. 2009 Multiscale geometric analysis of turbulence by curvelets. Phys. Fluids 21, 075104.

Marusic, I. 2001 On the role of large-scale structures in wall turbulence. Phys. Fluids 13, 735-743.

Marusic, I., McKeon, B. J., Monkewitz, P. A., Nagib, H. M., Smits, A. J. \& Sreenivasan, K. R. 2010 Wall-bounded turbulent flows at high Reynolds numbers: recent advances and key issues. Phys. Fluids 22, 065103.

MoIN, P. \& Kim, J. 1982 Numerical investigation of turbulent channel flow. J. Fluid Mech. 118, 341-377.

MoIn, P. \& KIm, J. 1985 The structure of the vorticity field in turbulent channel flow. Part 1. Analysis of instantaneous fields and statistical correlations. J. Fluid Mech. 155, 441-464.

Moser, R. D., Kim, J. \& Mansour, N. N. 1999 Direct numerical simulation of turbulent channel flow up to $R e_{\tau}=590$. Phys. Fluids 11, 943-945.

Okamoto, N., Yoshimatsu, K., Schneider, K., Farge, M. \& Kaneda, Y. 2007 Coherent vortices in high resolution direct numerical simulation of homogeneous isotropic turbulence: a wavelet viewpoint. Phys. Fluids 19, 115109.

ONG, L. \& Wallace, J. M. 1998 Joint probability density analysis of the structure and dynamics of the vorticity field of a turbulent boundary layer. J. Fluid Mech. 367, 291-328. 
Panton, R. L. 2001 Overview of the self-sustaining mechanisms of wall turbulence. Prog. Aerosp. Sci. 37, 341-383.

Perry, A. E. \& Chong, M. S. 1982 On the mechanism of wall turbulence. J. Fluid Mech. 119, $173-217$.

Perry, A. E., Henbest, S. \& Chong, M. S. 1986 A theoretical and experimental-study of wall turbulence. J. Fluid Mech. 165, 163-199.

Perry, A. E. \& Marusic, I. 1995 A wall-wake model for the turbulence structure of boundary-layers. Part 1. Extension of the attached eddy hypothesis. J. Fluid Mech. 298, 361-388.

Pope, S. B. 2000 Turbulent Flows. Cambridge University Press.

Pullin, D. I. 1981 The non-linear behavior of a constant vorticity layer at a wall. J. Fluid Mech. 108, 401-421.

Robinson, S. K. 1991 Coherent motions in the turbulent boundary layer. Annu. Rev. Fluid Mech. 23, 601-639.

Spalart, R. R., Moser, R. D. \& Rogers, M. M. 1991 Spectral methods for the Navier-Stokes equations with one infinite and two periodic directions. J. Comput. Phys. 96, 297-324.

Theodorsen, T. 1952 Mechanism of turbulence. In Proceedings of the Second Midwestern Conference on Fluid Mechanics, Ohio State University, Columbus, 17-19 March, pp. 1-18.

Townsend, A. A. 1976 The Structure of Turbulent Shear Flow, 2nd edn. Cambridge University Press.

Wallace, J. M., Brodkey, R. S. \& Eckelman, H. 1972 Wall region in turbulent shear flow. J. Fluid Mech. 54, 39-48.

Wu, X. \& MorN, P. 2009 Direct numerical simulation of turbulence in a nominally zero-pressuregradient flat-plate boundary layer. J. Fluid Mech. 630, 5-41.

YANG, Y. \& PUlLIN, D. I. 2010 On Lagrangian and vortex-surface fields for flows with Taylor-Green and Kida-Pelz initial conditions. J. Fluid Mech. 661, 446-481.

YAng, Y., Pullin, D. I. \& Bermejo-Moreno, I. 2010 Multi-scale geometric analysis of Lagrangian structures in isotropic turbulence. J. Fluid Mech. 654, 233-270.

Ying, L., Demanet, L. \& CANDÈs, E. 2005 3D discrete curvelet transform. In Proceedings of the Society of Photo-optical Instrumentation Engineers (SPIE), vol. 5914, pp. 351-361. SPIE.

Zhou, J., Adrian, R. J., Balachandar, S. \& Kendall, T. M. 1999 Mechanisms for generating coherent packets of hairpin vortices in channel flow. J. Fluid Mech. 387, 353-396. 